\title{
Single-molecule analysis of the entire perfringolysin $O$ pore formation pathway
}

Conall Mc Guinness ${ }^{1, \#}$, James C. Walsh ${ }^{1, \#, *}$, Charles Bayly-Jones ${ }^{2}$, Michelle A. Dunstone $^{2}$, Michelle Christie $^{3}$, Craig J. Morton ${ }^{3}$, Michael W. Parker ${ }^{3,4}$, Till Böcking ${ }^{1, *}$

${ }^{1}$ EMBL Australia Node in Single Molecule Science, School of Medical Sciences, Sydney, New South Wales, Australia

${ }^{2}$ Biomedicine Discovery Institute, Department of Biochemistry and Molecular Biology, Monash University, Melbourne, Victoria, Australia.

${ }^{3}$ Department of Biochemistry and Pharmacology, Bio21 Molecular Science and Biotechnology Institute, University of Melbourne, Victoria, Australia.

${ }^{4}$ Structural Biology Unit, St. Vincent's Institute of Medical Research, Victoria, Australia

\#equal contribution

${ }^{*}$ Corresponding authors: James Walsh (james.walsh@unsw.edu.au); Till Böcking

(till.boecking@unsw.edu.au) 


\begin{abstract}
The cholesterol-dependent cytolysin perfringolysin O (PFO) is secreted by Clostridium perfringens as a bacterial virulence factor able to form giant ring-shaped pores that perforate and ultimately lyse mammalian cell membranes. To resolve the kinetics of all steps in the assembly pathway, we have used single-molecule fluorescence imaging to follow the dynamics of PFO on dye-loaded liposomes that lead to opening of a pore and release of the encapsulated dye. Formation of a long-lived membrane-bound PFO dimer nucleates the growth of an irreversible oligomer. The growing oligomer can insert into the membrane and open a pore at stoichiometries ranging from tetramers to full rings ( 35 -mers), whereby the rate of insertion increases linearly with the number of subunits. Oligomers that insert before the ring is complete continue to grow by monomer addition post insertion. Overall, our observations suggest that PFO membrane insertion is kinetically controlled.
\end{abstract}




\section{Introduction}

Pore-forming proteins (PFPs) possess an ancient and ubiquitous mechanism for forming aqueous channels in the membranes surrounding cells and organelles (Dal Peraro and van der Goot, 2016; Johnstone et al., 2021). The largest and most sequence diverse class of PFPs is the membrane attack complex-perforin (MACPF)/cholesterol dependent cytolysin (CDC) superfamily with thousands of members now identified (Christie et al., 2018; Dunstone and Tweten, 2012; Rosado et al., 2008). Members of the MACPF family are found in all kingdoms of life but are most well characterised as effectors in the vertebrate immune system. Conversely, CDCs are bacterial virulence and defence factors (Tweten, 2005). While these two families differ greatly in sequence, they are linked together by a highly conserved 3D fold, which drives oligomerisation into rings of 12-40 PFP monomers and subsequent membrane insertion, ultimately forming a membrane-spanning $\beta$-barrel (Shatursky et al., 1999). The open pore has an unusually large lumen of $\sim 25-30 \mathrm{~nm}$ in the case of CDCs, allowing the passive transport of folded proteins across membranes.

Perfringolyin O (PFO), a prototypical example of a CDC, is secreted by the anaerobic bacterium Clostridium perfringens (Tweten et al., 2001), which is involved in the development of gas gangrene and necrohemorrhagic enteritis (Awad et al., 2001; Verherstraeten et al., 2015, 2013). As with the majority of CDCs, PFO binds to cholesterol-rich membranes and oligomerises to form large (25-30 nm) doughnut shaped pores, ultimately leading to cell lysis (Dang et al., 2005; Tilley et al., 2005). A small subset of CDCs do not require cholesterol for binding (Giddings et al., 2004; Ragaliauskas et al., 2019); however, it remains necessary for membrane insertion (Jacobs et al., 1998; Polekhina and Giddings, 2005).

The steps in canonical CDC pore-formation have been well characterised; the proteins are secreted as soluble monomers, monomers bind to and then oligomerise on target membranes to form a ring-shaped prepore complex, the prepore complex then undergoes a concerted conformational change and inserts in the membrane to form a large $(25-30 \mathrm{~nm})$ amphipathic $\beta$-barrel bilayer-spanning pore (Figure 1A) (Morton et al., 2019). To resolve these steps, point mutations of PFO have been used to investigate kinetically trapped intermediates alongside fluorescent conjugates acting as environmental indicators (Evans and Tweten, 2021; Ramachandran et al., 2004), with some intermediates observed by atomic force microscopy (AFM) (Czajkowsky et al., 2004) and electron microscopy (EM) (van Pee et al., 2017) high resolution imaging. These and other studies have defined the key molecular events and rearrangements required for pore formation. Initially, membrane binding is mediated by domain 4 (D4) which specifically recognises cholesterol in the lipid bilayer. Interdomain contacts in the CDC fold drive oligomerisation forming arc prepores. CDCs undergo a drastic collapse by rotation of domain 2 (D2) which lowers domain 3 (D3) toward the membrane, enabling the $\alpha$-helical bundles $(\alpha \mathrm{HB})$ to unfurl and insert into the bilayer forming transmembrane $\beta$-hairpins. Notably these data showed the prevalence of full prepore and inserted rings and assumed them to be the functional mechanism of pore formation, although incomplete arc-shaped oligomers have been observed by some labs (Leung et al., 2014; Sonnen et al., 2014). 
Liposome dye release assays have long been used to investigate the kinetics of PFO pore formation (Evans et al., 2020; Heuck et al., 2000; Shepard et al., 2000). This assay measures the rate of release of a fluorescent dye from liposomes in bulk after incubation with PFO, which is correlated to the rate of pore formation. The main shortcoming of these experiments is that they give a single bulk readout of the reaction over time. The intrinsically stochastic nature of nucleation makes it impossible to synchronise pore formation on multiple liposomes even if they are exposed to PFO at the same time. As a result, any ensemble measurement of PFO binding is blurred by averaging the growth of pores at different stages of formation. Similarly, pore insertion is the culmination of multiple stochastic processes, occurring asynchronously between liposomes. As such, it is difficult to identify the specific molecular interaction in which variation underpins any observed changes in the bulk measurement.

To overcome the limitation of ensemble averaging, imaging methods have been developed to follow PFP assembly at the level of individual pores (Ros et al., 2021; Sathyanarayana et al., 2018). Imaging modalities applied to CDC assembly on planar lipid bilayers include high speed atomic force microscopy, as shown for suilysin (Leung et al., 2014) and listeriolysin O (Ruan et al., 2016). In an accompanying paper, Wallace and colleagues use single-molecule fluorescence tracking of PFO assembly on a droplet interface bilayer (Senior et al., 2021). These imaging studies support the insertion of incomplete arc-shaped membrane lesions, suggesting an alternative mechanism of pore formation that is distinct from the canonical prepore formation prior to insertion. This discrepancy raises the question as to when and how release of the membrane spanning regions is triggered, which cannot be correlated with key assembly steps using ensemble methods. Related to this matter, it is also unclear whether release and insertion of the membrane spanning regions from each of the subunits occurs in a concerted or sequential fashion.

To observe PFP assembly and membrane permeabilisation simultaneously at the single molecule level, we have adapted the liposome dye release assay to measure kinetics on individual liposomes. By using single molecule total internal reflection fluorescence (TIRF) microscopy, we visualised the binding, nucleation, build up and insertion of individual PFO species. These data were subsequently used to develop a mathematical model for PFO pore formation in which parameters can predict the number of subunits in PFO oligomers at the time of insertion. We have also found in our assay that inserted PFO arcs, currently thought to be kinetically trapped, can continue to grow post insertion. 


\section{Results}

\section{Fluorescence imaging of PFO pore assembly kinetics on single liposomes}

Here we developed a single-molecule approach to observe in real time the dynamic interactions between PFO and dye-loaded liposomes leading to dye release from individual liposomes using total internal reflection fluorescence (TIRF) microscopy. The assay design is shown schematically in Figure 1A. Large unilamellar liposomes with an average diameter of $\sim 200 \mathrm{~nm}$ (Figure 1-Figure Supplement 1) made of a synthetic lipid mixture containing cholesterol, 1-palmitoyl-2-oleoyl-phosphatidylcholine and a small amount of a phosphatidylethanolamine derivative with biotinylated headgroup (55:44:1 molar ratio) were loaded with the small fluorescent dye Alexa Fluor 488 (AF488) during extrusion. The liposomes were captured on the surface of a streptavidin-coated glass coverslip at the bottom of a microfluidic channel device. Recombinant cysteine-less PFO (see Appendix) was labelled via lysine residues (Harris et al., 1991) with Alexa Fluor 647 (AF647) using NHS ester chemistry, whereby most molecules contained a single dye (Figure 2E) and AF647-PFO retained full pore-formation activity (Figure 1-Figure Supplement 2). AF647-PFO was constantly flowed at a defined concentration through the microfluidic channel while imaging AF647-PFO assembly and AF488 release from liposomes by dual-colour time-lapse TIRF microscopy. The immobilised liposomes appeared as diffraction-limited spots in the AF488 channel (Figure 1B, top left), which disappeared over the course of the experiment (Figure 1B, bottom left). In contrast, AF647-PFO was initially undetectable (Figure 1B, top middle), before gradually accumulating in spots that colocalised with the liposomes (Figure 1B, middle bottom). Liposomes subjected to the same buffer flow, but in the presence of AF647-PFO concentrations ( $\leq 20 \mathrm{pM})$ below the threshold required for PFO assembly, retained the content dye for at least 7 hours, confirming that dye release was dependent on pore formation (Figure 2-Figure Supplement 2).

TIRF pore formation movies were analysed using automated software for tracking the fluorescence intensity over time at each liposome location in both channels to generate single-liposome AF647-PFO binding and content dye release traces. A typical dual-colour pore formation trace recorded at a single liposome (highlighted with a white box in Figure 1B) is shown in Figure 1C. A montage of corresponding images of the liposome and AF647-PFO channels are shown below the plot. Initially, the liposome intensity remains high while there is no signal above noise in the AF647-PFO channel. During this phase, PFO monomers interact transiently with the liposome membrane, which can be resolved by imaging at high temporal resolution (as described below) but are not detected in the pore formation traces. Eventually, the AF647-PFO signal rises above background, which we attribute to nucleation of a PFO oligomer that is stably bound to the liposome membrane and continues to grow in length (and hence intensity). A sudden drop in the content dye signal to background levels pinpoints the time of membrane poration, allowing rapid diffusion of AF488 out of the liposome. We attribute this event to the opening of a transmembrane pore as a result of PFO oligomer insertion into the membrane. We also determined the number of labelled subunits in the PFO oligomer forming the open pore structure from the AF647 intensity at the time of dye release divided by the intensity of a single AF647-PFO molecule. Interestingly, the AF647-PFO signal continues to increase beyond the time of pore formation on most liposomes. When experiments were 
continued long after poration, liposomes continued to bind PFO to levels much greater than the value expected for a single ring-shaped pore ( $\sim 35$ subunits), suggesting that eventually multiple pores form on a single liposome. Further example traces are shown in Figure 1 Figure Supplement 3.

Typically, a field of view contained $\sim 2300$ liposomes (corresponding to a surface density of 0.074 liposomes $/ \mu^{2}{ }^{2}$ ). At AF647-PFO concentrations between 100-500 pM, most liposomes lost their dye signal in a single step (>50\%), while $\sim 30 \%$ of dye release traces showed partial or multi-step signal loss (Figure 1D). The remainder ( 10\%) showed no or little signal loss (Figure 1D), suggesting that these were not permeabilised despite AF647-PFO binding to many of these liposomes (Figure 1 Figure Supplement 3B). At 50 pM AF647-PFO, single-step pore formation was less efficient ( $<10 \%)$, and at even lower AF647-PFO concentrations, dye release was no longer observed (Figure 2-Figure Supplement 2). We tentatively attribute the different release profiles to heterogeneity in the liposome preparations. For example, aggregated liposomes and multilamellar liposomes would be expected to give rise to traces with multiple steps or partial release of dye. Single-step traces were included in further analysis and initial inspection revealed that the time for nucleation of a growing PFO oligomer and the time for poration varied between liposomes, as did the number of subunits at the time of pore opening. These processes are analysed in more detail below. As expected, the kinetics of liposome poration decreased with increasing AF647-PFO concentration (Figure 1E). At high concentrations (500 pM, Figure 1E, light blue) single-step dye release was complete within $\sim 20$ min, while at low concentrations ( $50 \mathrm{pM}$, Figure 1E, dark blue) this process takes $\sim 2$ hours.

\section{Characterisation of PFO monomer binding to liposomes}

The first step of PFO pore formation involves the binding of PFO monomers to the membrane (Figure 1A, left). To measure the kinetics of this process, we imaged the interactions of AF647-PFO molecules with liposomes at high temporal resolution ( 17.5 frames/s) for a period of 7 hours at room temperature at very low concentrations $(2.5-20 \mathrm{pM})$, where the chance of two monomers binding to a liposome at the same time to dimerise becomes negligible. Under these conditions, we observed transient binding of single AF647-PFO molecules appearing as sporadic fluorescent spots. We then used a software developed for single-molecule localisation microscopy (Schnitzbauer et al., 2017) to detect and track AF647-PFO spots appearing and disappearing at the locations of individual liposomes, resulting in a trace at each location with typically over 1000 binding events. A representative single-liposome trace recorded in the presence of 10 pM AF647-PFO shows that the rate of binding events remained constant over the entire 7 hour experiment (Figure 2A), while zoomed-in views show sporadic binding intensity spikes (Figure 2B) corresponding to a AF647-PFO signal persisting for several frames (Figure $2 \mathrm{C}$ ). The intensity distribution of all binding events on all liposomes overlaid completely with the intensity distribution of the same batch of AF647-PFO irreversibly immobilised directly on a glass coverslip (Figure 2D). Single-molecule photobleaching confirmed that most molecules (84\%) bleached in a single step (Figure 2E). Taken together, these observations demonstrate that AF647-PFO binds transiently to liposomes as monomers. 
The scatter plot of $\mathrm{x} / \mathrm{y}$-coordinates for all molecules detected in Figure $2 \mathrm{~A}-\mathrm{C}$ forms a ring-like distribution, as expected for the z-projection of AF647-PFO molecules binding to the membrane of a spherical liposome (Figure 2F). The $x / y$-coordinate maps for all liposomes were further used to reconstruct a super-resolved image, which revealed the outlines of liposomes bound to the coverslip (Figure 2G). Interestingly, many liposomes displayed bright spots in the reconstruction images suggesting that liposome membranes may have a spatially inhomogeneous affinity for binding PFO, e.g. due to differences in cholesterol distribution. Importantly, the super-resolved AF647-PFO structures colocalised with the diffraction-limited signals in the AF488 content dye channel, confirming that binding occurred on liposomes (Figure 2G/H and Figure 2-Figure Supplement 1).

Next, we determined the rates of AF647-PFO monomer binding and unbinding from each binding trace collected at a single liposome. First, we measured the time intervals between peaks in the trace (an example of one such $\Delta t_{\text {dark }}$ is highlighted in Figure $2 \mathrm{~B}$ ) to determine the distribution of waiting times before binding of the next molecule (Figure 2I). An exponential fit of this distribution provided the AF647-PFO monomer binding rate. Similarly, we measured the duration of each peak (an example of one such $\Delta t_{\text {bright }}$ is highlighted in Figure $2 \mathrm{C}$ ) to determine the time distribution of the bound state (Figure $2 \mathrm{~L}$ ). An exponential fit of this distribution then provided the AF647-PFO monomer unbinding rate. We repeated this analysis for all liposomes in the field of view, revealing a wide distribution of binding and unbinding rates on different liposomes (Figure $2 \mathrm{~J}$ and $\mathrm{M}$, respectively), possibly due to inhomogeneity in binding affinities of different structural states and/or local lipid composition. Analysis of binding experiments at a range of AF647-PFO concentrations showed that the binding rate distributions shifted to higher values with concentration (Figure $2 \mathrm{~J}$ ), whereby the median monomer binding rate increased linearly with concentration (Figure $2 \mathrm{~K}$ ). This analysis allowed us to obtain the monomer binding rate constant $\left(B=0.9 \mathrm{nM}^{-1} \mathrm{~s}^{-1}\right.$, corresponding to $7.2 \mathrm{nM}^{-1} \mathrm{~s}^{-1} \mu \mathrm{m}^{-2}$ when taking the surface area of $200 \mathrm{~nm}$ liposomes into account). As expected, the unbinding rate distributions (Figure 2M) and the median unbinding rates obtained from these distributions were independent of concentration (Figure 2N), yielding a value for the unbinding rate of $U=2.27 \mathrm{~s}^{-1}$.

Taken together, our observations show that single AF647-PFO molecules rapidly cycle between the solution and the membrane, whereby the membrane-bound state is short-lived (half-life of $0.3 \mathrm{~s}$ ). As expected, these interactions did not lead to pore formation, as liposomes retained their content dye for the duration of the experiments (Figure 2-Figure Supplement 2).

\section{PFO dimerisation on the membrane produces a metastable complex}

The fast frame rate and high laser power required to image the binding of single AF647-PFO molecules to liposomes (Figure 2) made it impossible to observe longer-lived species as they would be rapidly photobleached. TIRF binding experiments with a much slower frame rate (3 frames per minute) and lower laser power allowed us to detect rare long-lived species (1063 events from $\sim 7500$ liposomes viewed over 11 hours) as illustrated by the trace with an AF647-PFO signal that persisted for $\sim 20 \mathrm{~min}$ (Figure 3A/B). This signal colocalised with an AF488-loaded liposome (Figure 3C/D), consistent with binding to the membrane. 
To determine the stoichiometry of AF647-PFO in the long-lived state, we measured the average intensity of all long-lived signals on liposomes. The resulting intensity distribution showed that the species was almost twice as bright as monomeric AF647-PFO on glass and agreed more closely with the predicted intensity distribution (Mutch et al., 2007) of a dimer (Figure 3E). To determine the dissociation kinetics of this species, we measured the duration of all long-lived signals. The resulting distribution decayed exponentially (Figure 3F), suggesting that signal disappearance is governed by a single step with a rate of $D_{\mathrm{r}}=0.052 \mathrm{~min}^{-1}$ (after correction for photobleaching). Finally, we tested whether even longer-lived species could be detected by further decreasing the frame rate ( 0.5 frames per minute), but only recovered the same dimer species described above (Figure 3-Figure Supplement 1).

Taken together, this analysis shows that at low concentrations, the interactions of AF647-PFO on liposomes very rarely lead to the formation of a metastable dimer on the membrane that persist with a half-life of $13 \mathrm{~min}$ (corresponding to a mean lifetime of $20 \mathrm{~min}$ ), i.e. three orders of magnitude longer than the monomer. We conclude that dimerisation on the membrane promotes strong PFO-PFO and PFO-membrane interactions. From our data we cannot distinguish whether the dimer disappears by unbinding from the membrane, or whether it dissociates to monomers that subsequently unbind from the membrane. We also note that the half-life of the dimer state is sufficiently long such that it is essentially irreversible on the time scale of PFO assembly at concentrations of $\geq 100 \mathrm{pM}$, where the entire pore formation process takes on average less than 20 min (Figure 1E).

\section{PFO dimerisation on the membrane nucleates a stably growing oligomer}

At higher concentrations ( $\geq 25 \mathrm{pM}$ ), the transient interactions of PFO eventually lead to nucleation of a membrane-bound oligomer that continues to grow (Figure 1C and Figure 4A-C). The nucleation times determined from AF647 traces recorded at concentrations between 100-500 pM followed single exponential distributions (Figure 4D/E, gamma shape parameters $\approx 1$ ), suggesting that nucleation is governed by a single rate-limiting step. The nucleation times at $50 \mathrm{pM}$ were better described by a gamma distribution with a shape factor of $\sim 2$ (Figure 4D/E), suggesting that at very low PFO concentrations close to the threshold where nucleation is no longer observed, nucleation is also governed by an additional rate-limiting step. The nucleation rate determined from the exponential fit of the nucleation time distribution increased with AF647-PFO concentration (Figure 4F, Figure 4-Figure Supplement 1); at higher concentrations it becomes more probable for a second PFO molecule to bind to the membrane before the first one has fallen off.

To determine the post-nucleation kinetics of AF647-PFO oligomerisation, we generated the mean AF647-PFO intensity trace by aligning all traces recorded at single liposomes at the time of nucleation. The mean traces obtained for a range of concentrations (Figure 4B) show that oligomerisation occurs at a constant rate (all lines are linear) that increases with concentration. To further quantify the dependence of oligomerisation on concentration, we fitted the steady increase in fluorescence intensity after nucleation for each liposome with a linear function, whereby the slope provided the oligomerisation rate. As expected, the median oligomerisation rate for all liposomes increased linearly with AF647-PFO concentration (Figure 4C), providing an 
oligomerisation rate constant of $P=0.23 \mathrm{nM}^{-1} \mathrm{~s}^{-1}$. This oligomerisation rate is essentially the product of the rate of PFO monomer binding to the membrane and the probability of finding and joining the growing oligomer. The ratio between the oligomerisation rate constant $\left(0.23 \mathrm{nM}^{-1} \mathrm{~s}^{-1}\right)$ to the single molecule binding rate constant $\left(0.9 \mathrm{nM}^{-1} \mathrm{~s}^{-1}\right)$ reveals that $25 \%$ of the time, a PFO monomer binding to the membrane will end up joining the oligomer.

The oligomer disassembly rate (given by the Y-intercept of the fit line in Figure 4C) is effectively zero suggesting that PFO oligomers are stable on the membrane and do not dissociate. We conclude that PFO oligomerisation is essentially irreversible on the time scale of pore formation. The high stability of PFO oligomers on the membrane is also observed after removal of AF647-PFO from solution (Figure 5-Figure Supplement 1C), as discussed below, and is not surprising given the long mean lifetime of the dimer on the membrane (20 minutes).

On the basis of the high dimer stability, we reasoned that the dimer represents the stable nucleus for oligomerisation, i.e. the rate-limiting step for nucleation is waiting for two monomers to be bound to the membrane at the same time and form a dimer. We used the kinetic model shown in the inset of Figure 4F to calculate theoretical nucleation rates, whereby firstly, membrane binding is governed by the binding and unbinding kinetics of monomers (Figure 2) and secondly, dimerisation on the membrane is governed by a dimerisation rate constant, $D_{\mathrm{f}}$. We fitted the model to the experimental concentration dependence of nucleation rates (Figure $4 \mathrm{~F}$ ) with $D_{\mathrm{f}}$ as the only free parameter to obtain an estimate of $D_{\mathrm{f}}=0.16 \mathrm{nM}^{-1} \mathrm{~s}^{-1}$, similar to the value for the oligomerisation rate constant obtained above $\left(P=0.23 \mathrm{nM}^{-1} \mathrm{~s}^{-1}\right)$. Thus, dimerisation is kinetically similar to oligomerisation, consistent with the dimer being the first stable species on the membrane. Accordingly, the predicted nucleation rates when using the value of $P$ to parametrise dimerisation in the model were also in reasonable agreement with the experimental data. This agreement is remarkable, given that parameter values for the monomer binding/unbinding and oligomerisation kinetics were obtained using different experimental conditions and are sufficient to predict nucleation rates.

Finally, we extended the kinetic model to account for the finite dimer stability measured in Figure 3. Dimer dissociation may occur via one of two alternative pathways, i.e. unbind from the membrane or fall apart into monomers. Fits of these extended models to the experimental concentration dependence of nucleation rates were essentially indistinguishable from the simple model described above (Figure 4-Figure Supplement 2). This is because the dimer dissociation process is so slow, that it has virtually no impact on the outcome of nucleation in the concentration range investigated here, regardless of the pathway.

\section{PFO membrane insertion kinetics increase with oligomer length}

Dual-colour pore formation traces showed that membrane pore opening (dye release) often occurred soon after onset of the AF647-PFO signal increase (Figure 1 Figure Supplement 3), and analysis of the number of subunits at the time of dye release showed that low stoichiometry PFO oligomers (interpreted as arcs) insert into the membrane to form pores before they form full rings. The pore opening kinetics of continuously growing arcs is complicated and analysed in detail below. To first measure (arc) pore opening kinetics in the absence of continuing 
oligomerisation, we devised a wash-out assay, in which liposomes are exposed to AF647-PFO for a limited time to allow formation of membrane-bound arcs. The AF647-PFO was then washed out of the microfluidics channel using a buffer at a time when the majority of liposomes still retained their content dye (i.e. the pore had not yet formed).

After wash-out, we observed that these dye-loaded liposomes showed a wide distribution of AF647-PFO oligomer lengths between 2-40 subunits (Figure 5-Figure Supplement 1A), as expected for a stochastic nucleation and growth process. We then continued to image the liposomes by TIRF microscopy to detect the dye release as a read-out for pore formation. A typical example trace recorded at a single liposome is shown in Figure 5A. Initially, AF647-PFO nucleated a growing arc (signal appearance and increase) on the membrane, while the content dye signal remained high. After wash-out, the AF647-PFO signal stayed constant for the remainder of the experiment, confirming that membrane-bound arcs did not release PFO subunits (see also Figure 5-Figure Supplement 1C). After a waiting time, the content dye signal disappeared, which we interpret as the insertion of the PFO arc into the membrane and concomitant opening of the membrane pore. Thus, we defined the time period between wash-out and dye release as the insertion time. Since our experimental read-out (dye release) reports the opening of the membrane pore, this definition assumes that the rearrangement of lipids required to open the semi-toroidal membrane pore is fast compared to time required to wait for PFO insertion.

When applied to all liposomes, this analysis allowed us to determine the efficiency and kinetics of pore formation for arcs containing a defined number of subunits (oligomer length). First, only a low fraction of liposomes with AF647-PFO dimers or trimers released their content dye, but pore formation efficiency increased sharply with oligomer length, reaching half-maximal efficiency for tetramers and becoming length-independent for arcs containing at least 6 subunits (Figure 5B). We conclude that arcs with at least 4 subunits could efficiently insert into the membrane resulting in the opening of a transmembrane pore, as observed in molecular dynamics simulations of a membrane-inserted CDC pentamer (Vögele et al., 2019). Second, we extracted the insertion time distributions for each oligomer length. These distributions could be described with a single exponential function to yield the oligomer-length specific insertion rate (assumed to be the rate-limiting step for pore opening). Surprisingly, the insertion rate increased linearly with oligomer length (Figure 5C), from species corresponding to tetramers to full rings. Taken together, these observations suggest a stochastic trigger for the prepore to pore transition as discussed in more detail below.

\section{Membrane insertion represents the main energy barrier for pore formation}

We repeated the wash-out experiments at a range of temperatures to determine the activation energy of PFO oligomer insertion. First, we extracted the oligomer-length dependence of insertion rates between $22-37^{\circ} \mathrm{C}$ (Figure 5D). Fitting of the relative activities at each temperature provided an activation energy of $26.5 \mathrm{kcal} \mathrm{mol}^{-1}$ for the transition from the prepore to the open pore state; this analysis is shown as an Arrhenius plot in Figure 5E. For comparison, the activation energy determined from ensemble measurements for the entire pore formation 
pathway are similar (23.9 \pm 1.3 (Wade et al., 2015) or $28 \pm 1.9 \mathrm{kcal} \mathrm{mol}^{-1}$ (Wade et al., 2019)). We conclude that the insertion event is the dominant energy barrier during pore formation.

\section{Pore insertion kinetics for continuously growing arcs}

The dual-colour pore formation experiments showed that even in the continued presence of AF647-PFO in solution, arcs instead of full rings were the most common species to perforate the liposome membrane. To obtain an estimate of the insertion rate as a function of the number of subunits in the case of a continuously growing arc, we defined a normalised rate as follows. We divided the total number of insertion events for each given oligomer length by the cumulative observed time across all detected instances of AF647-PFO oligomers of that length. As observed in the wash-out experiments above, the insertion rate depended linearly on the oligomer length at AF647-PFO concentrations between 50-500 pM (Figure 6A). Surprisingly, the insertion rate also increased linearly with the AF647-PFO concentration in solution (Figure 6A and Figure 6-Figure Supplement 1).

To predict the oligomer lengths and kinetics of insertion, we developed a kinetics model (Figure $6 \mathrm{~B}$, explained in detail in the Appendix) that is entirely parameterised by the experimentally determined rates for oligomerisation and insertion. In this model, the assembly process starts from a stable dimer nucleus. Below the minimum arc length required for efficient insertion and pore opening $(n<4)$, the arc grows by monomer addition. Once the oligomer reaches $n=4$, it can either grow or insert, whereby the probability of growth versus insertion is determined by the respective rates for these processes.

Next, we validated the model by comparing predictions to experimentally determined distributions of intermediates detectable in the dual colour pore formation experiments. The lengths of oligomers inserting into the membrane, determined as the number of subunits from the AF647-PFO signal at the time of dye release, was broadly distributed at all AF647-PFO concentrations (Figure 6-Figure Supplement 2A), whereby the upper end of the distributions represented the level expected for full rings ( 35 subunits (Czajkowsky et al., 2004; Dang et al., 2005)). The mean oligomer length (Figure 6C) increased with concentration from fewer than 15 subunits at $50 \mathrm{pM}$ to 20 subunits at $500 \mathrm{pM}$. Similarly, the fraction of oligomers inserting at a length consistent with a complete ring increased with concentration (Figure 6D) but remained below $30 \%$ even at the highest concentrations tested here. This outcome reflects that the oligomerisation rate was on average not fast enough to complete the full ring before arc insertion occurred. The kinetic model faithfully reproduced the concentration dependence of length distributions (Figure 6-Figure Supplement 2), mean lengths (Figure 6C, orange line) and predicted the fraction of complete rings (Figure 6D, orange bars) at insertion within a factor of two.

From the single-liposome traces we were also able to measure the time required from nucleation to opening of the membrane pore (Figure 6E). On average, this time decreased with AF647-PFO concentration, as expected given that arcs grow more quickly at higher concentration and longer arcs insert more quickly. This trend was also correctly predicted by the 
model (Figure 6E, orange line). Overall, the excellent agreement between data and model predictions supports the dependence of insertion on PFO oligomer length and concentration.

\section{PFO arcs continue to grow after insertion}

Next, we analysed the AF647-PFO signal before and after the transition of the arc in the prepore state to the pore state had occurred (Figure 6F). It has previously been shown that arcs of the related CDC suilysin do not continue to grow after the transition from the prepore to the pore state (Leung et al., 2014). Surprisingly, the AF647-PFO oligomerisation rate was the same immediately after poration (dye release) (Figure 6F, yellow symbols) as it was just before poration (Figure 6F, orange symbols), i.e. the AF647-PFO signal increase did not stop (or pause) before or after insertion. Since the nucleation of a new structure is a slow process that would lead to a pause in the AF647-PFO signal increase, we interpret this observation as the continued addition of monomers (that bind from solution to the membrane) to the arc pore. An alternative explanation, i.e. the simultaneous growth of a second oligomer on the liposome, is unlikely for the following reasons: The nucleation of a second oligomer before insertion is unlikely since the first oligomer acts as a sink for monomers, further slowing an already slow nucleation step. Also, the appearance of additional oligomers would be apparent from an increase in the oligomerisation rate, which is not observed in our data (Figure 6F, orange symbols). This interpretation of nucleating a single oligomer per liposome is also consistent with negative staining EM images of PFO structures which appear as single complete rings, despite being assembled at relatively higher (nanomolar) concentrations (Figure 6-Figure Supplement 4).

\section{High pH inhibits PFO pore formation activity at the step of membrane binding}

Above $\mathrm{pH}$ 7, PFO accumulation on membranes and pore formation is impaired (Nelson et al., 2008), but it remains unclear where in the pathway the defect occurs. Stable PFO accumulation on membranes requires oligomerisation, which depends on interactions with the membrane as well as protein-protein interactions. To dissect which interactions and whether specific steps in the PFO pore formation pathway are affected by $\mathrm{pH}$, we used the dual-colour pore formation assay to measure assembly kinetics of AF647-PFO (200 pM) and dye release from liposomes between $\mathrm{pH}$ 5-8 in conjunction with kinetic modelling (Figure 7). Permeabilisation of liposomes was most efficient at $\mathrm{pH} \leq 6.5$ and then rapidly diminished between $\mathrm{pH} 7-8$ (Figure 7A), whereby $<3 \%$ of liposomes showed single-step dye release characteristic of pore formation at $\mathrm{pH}$. We note that the pore formation efficiency was lower in these experiments than above (the fraction of no dye release traces is $34 \%$ in Figure $7 \mathrm{~A}$ vs. $13 \%$ in Figure 1D), possibly due to differences between batches of AF647-PFO. In addition to $\mathrm{pH}$ dependence of pore formation efficiency, the overall rate of pore formation slows down with increasing $\mathrm{pH}$ (Figure 7B).

Next, we inspected oligomerisation (Figure 7C) and nucleation (Figure 7D), as expected we observed a reduction in rate over the $\mathrm{pH}$ range, whereby nucleation was almost abolished at $\mathrm{pH}$ 8 (Figure 7-Figure Supplement 1). This decrease in rate may result from either reduced membrane binding of PFO monomers or from reduced lateral interactions between PFO molecules forming the oligomer. To distinguish between these two possibilities, we used the kinetic model in Figure 4E to predict the effect of weakening either of these interactions on 
nucleation. The better fit of the data by the membrane binding scenario (Figure 7D, purple line) leads us to conclude the observed decrease in nucleation kinetics with $\mathrm{pH}$ was due to a defect in PFO-membrane binding rather than a defect in PFO-PFO interactions, whereby we estimate that the affinity of PFO monomers for membranes is decreased by 10 -fold between $\mathrm{pH} 5$ and $\mathrm{pH}$ 8.

It has been proposed that the transition from the prepore to the membrane-inserted state could be affected by $\mathrm{pH}$ (Rossjohn et al., 2007). To test this possibility, we extracted the insertion kinetics as a function of oligomer length as described above, which showed minor differences but no overall correlation between the insertion rate and $\mathrm{pH}$ (Figure 7E). This suggests that monomer binding was the only step affected by $\mathrm{pH}$.

Next, we analysed the phase from nucleation to poration. Interestingly, the number of subunits in the arc at the time of poration was higher at pH 5 ( $\sim 25$ subunits) than at $\mathrm{pH} 8$ ( 10 subunits) (Figure 7F) and similarly the fraction of PFO oligomers that insert as complete rings decreased from $\sim 30 \%$ to none detected across the $\mathrm{pH}$ range (Figure 7F). Concomitantly, the time from nucleation to poration increased with $\mathrm{pH}$ (Figure 7G). When we simply introduced the $\mathrm{pH}$-dependent differences in the oligomerisation rate (which in turn result from the defect in membrane binding) into the oligomerisation/insertion kinetic model (Figure 6B), the model was able to reproduce the decrease in mean arc size and complete ring formation (Figure 7F/G) and the increase in the time from nucleation to poration (Figure 7G) with increasing $\mathrm{pH}$. Thus, the kinetics and energetics of membrane insertion are not affected by $\mathrm{pH}$. Finally, we confirmed that across the entire $\mathrm{pH}$ range oligomerisation continued with unchanged kinetics after arc insertion, i.e. the basic features of the pore assembly pathway were preserved.

On the basis of these analyses we conclude that the overall pronounced decrease of the pore formation efficiency and kinetics with increasing $\mathrm{pH}$ from slightly acidic to slightly alkaline is due to reduced affinity of the monomer binding to the membrane while PFO-PFO interactions are largely unaffected. This defect propagates through other phases of the assembly pathway, slowing nucleation and oligomerisation but not the kinetics of the prepore to pore transition. As a consequence, the mean number of subunits at the time of poration decreases with increasing $\mathrm{pH}$. The implication of this observation is that other parameters that affect PFO-membrane affinity (such as lipid composition) would similarly tune whether insertion occurs early during PFO arc growth or when rings are (almost) complete. 


\section{Discussion}

By measuring the single-molecule kinetics of PFO accumulation on liposomes together with a functional read-out for pore opening at the level of single liposomes, we were able to identify distinct stages in the pore formation pathway that would be undetectable in ensemble measurements due to dephasing. Our analysis provides a complete kinetic description of CDC pore assembly from monomer binding to pore opening with the following characteristics: (i) PFO monomers repeatedly bind to and diffuse on the membrane surface and dissociate with a half-life of $0.3 \mathrm{~s}$; (ii) dimerisation of two PFO monomers on the membrane represents the committed step that nucleates a stable growing oligomer; (iii) oligomerisation occurs by addition of monomers that bind to the membrane from solution and is essentially irreversible; (iv) oligomers (arcs) with as few as four (possibly fewer) subunits can insert into the membrane and open a membrane pore, whereby the rate of insertion increases approximately linearly with the number of subunits in the arc; this transition from the prepore to the pore state constitutes the main energy barrier in the pathway; ( $v$ ) arcs continue to grow after insertion by addition of PFO monomers, presumably forming complete rings.

Our experimental approach relying on immobilised liposomes is complementary to AFM (Boyd et al., 2016; Leung et al., 2014; Mulvihill et al., 2015; Vögele et al., 2019) and single-molecule fluorescence microscopy (Senior et al., 2021) studies on flat bilayers to image the dynamic process of CDC pore assembly in real time. The assay has sufficiently high throughput to study the effect of varying experimental conditions on pore formation (in this work, PFO concentrations and $\mathrm{pH}$ ), whereby each experiment provides traces from hundreds to over a thousand liposomes providing statistical power to detect small changes in kinetic parameters. The use of microfluidics enables temporal control of experimental conditions. In addition, the constant microfluidic solution flow facilitates experiments at exquisitely low concentrations by avoiding depletion of PFO from solution due to adsorption onto surfaces and assembly on membranes. TIRF microscopy provides sensitivity for single-molecule detection at high temporal resolution (57 ms per frame) for prolonged periods of time (hours). This allowed us to measure the transient interactions of PFO monomers on liposomes at concentrations ( $\leq 20 \mathrm{pM})$ where dimerisation on the membrane surface is highly improbable, providing the first estimates of the monomer binding and unbinding rates for PFO.

Limitations of our approach include the following: unlike measurements on flat membranes, we cannot measure the lateral diffusion of molecules and complexes on the liposome membrane, nor can we distinguish separate oligomers on the same liposome due to the diffraction limit. Non-specific labelling of PFO (resulting in a heterogeneous sample of labelled species), as well as fluorescence artefacts, limit the precision with which we can determine the number of subunits in PFO arcs. To be able to observe the growth of a single complexes, the concentrations used in this study were limited to the $\mathrm{pM}$ range, while biochemical characterisation and AFM imaging of PFPs is typically carried out in the nM range.

Consistent with previous observations for PFO and other CDC/MACPF pore formers (Leung et al., 2017, 2014; Senior et al., 2021), we find that PFO oligomerisation is irreversible. Notably, 
our data also shows that the PFO dimer is the first long-lived species on the membrane, such that dimerisation is the committed step for pore formation. PFO does not form dimers (or oligomers) in solution (Feil et al., 1996; Rossjohn et al., 1997). On the membrane, collisions between PFO molecules may initially form weak, reversible interactions but stable PFO-PFO association is dependent on a conformational change that can only occur on the membrane surface (Hotze et al., 2012). This requires displacement of the $\beta 5$ strand from the $\beta$-sheet in domain 3 to allow interactions between the $\beta 4$ and $\beta 1$ ' strands of adjacent PFO molecules defining the nascent $\beta$-barrel (Evans et al., 2020; Ramachandran et al., 2004). It is worth noting that after oligomerisation, MACPF/CDC $\beta$-barrels are typically SDS-resistant and show remarkable stability (Evans et al., 2020; Heuck et al., 2000; Shepard et al., 2000). Our data only revealed one long-lived dimer species, suggesting that the proposed reversible dimer is too short-lived to be detected here and either dissociates or immediately converts to the stable dimer. The type of conformational change required for this transition is illustrated in Figure 2-Figure Supplements 3 and 4 and resembles the pathway for dimerisation proposed on the basis of linear CDC oligomers in pneumolysin, vaginolysin and intermedilysin crystal structures (Lawrence et al., 2016, 2015). Finally, the level of stabilisation observed here ( 3 orders of magnitude increase in half-life on the membrane) is remarkable even when accounting for avidity of a tight PFO-PFO dimer, suggesting that dimerisation goes along with further conformational changes to stabilise the membrane-bound state.

It has been shown that several MACPF/CDC PFPs can insert into the membrane as arcs before formation of the full ring is complete (Leung et al., 2014; Mulvihill et al., 2015; Podobnik et al., 2015; Sonnen et al., 2014). Our analysis showed that PFO oligomers with as few as four subunits can efficiently release the encapsulated dye from the liposome (Figure 5). Since PFO assembly on liposomes is not sufficient for dye release, but requires unfurling and insertion of the $\beta$-hairpins (Heuck et al., 2003, 2000), our data suggests that small oligomers can insert into the membrane and induce formation of a (at least transient) semi-toroidal pore. PFO dimers and trimers do not efficiently release dye (Figure 5), but we cannot distinguish whether these species fail to insert into the bilayer or fail to induce membrane pore opening upon insertion. Membrane-bound monomers are short-lived, rapidly dissociating back into solution (Figure 2), and thus do not insert into the lipid bilayer.

Remarkably, the rate of arc insertion increased linearly with the number of subunits in the arc such that insertion of low order oligomers can only be observed in the absence of growth. One interpretation of the oligomer length dependence is that any subunit can independently trigger the process leading to insertion of the entire arc, i.e. the more subunits are available, the higher the probability of insertion. Overall, our interpretation fits with the sequential insertion model for $\beta$-barrel formation (van Pee et al., 2017), whereby the conformational change in one subunit propagates to neighbouring subunits along the oligomer. In this model, the semi-toroidal pore opens early in the process and lipids are pushed aside into the bulk membrane as insertion proceeds along the oligomer (Vögele et al., 2019).

Our experiments also suggested that when oligomers are continuously growing, the insertion rate increases with the concentration of PFO in solution. This unexpected observation needs 
further investigation and validation. While we do not yet understand the underlying mechanism, one possible hypothesis is that the binding of a monomer to the growing oligomer induces a transient activated state that is more likely to insert. More frequent binding events (observed at higher PFO concentrations in solution) would then induce the activated state more frequently, leading to an overall increase in insertion kinetics.

We observed that AF647-PFO accumulation on the liposome continued unabated after opening of an arc pore, which we interpret as post-insertion oligomer growth. Using single-molecule tracking on droplet interface bilayers supported on an agarose layer, Wallace and colleagues in the accompanying paper also observed that PFO arcs continue to grow by monomer addition after insertion into the bilayer (Senior et al., 2021), whereby insertion was detected by the sudden drop in lateral diffusion of the assembling structure on the membrane. In contrast, AFM studies of suilysin pore formation on lipid bilayers supported on mica have shown that suilysin arcs do not continue to grow after insertion (Leung et al., 2014). Thus, further studies are needed to determine whether different CDCs and/or different experimental systems lead to different outcomes.

Real-time imaging (Leung et al., 2017; Parsons et al., 2019) and structural analysis (Spicer et al., 2018) suggest that some members of the MACPF family can assemble via a growing pore mechanism whereby monomers or oligomers add to an inserted arc. Notably, MACPFs do not undergo a vertical collapse upon insertion such that oligomerisation interfaces remain aligned between molecules in the prepore and the pore state. In contrast, it is unclear how membrane-bound PFO monomers in the prepore state could join a collapsed inserted arc and how this interaction could lead to insertion of the newly arrived PFO molecule since insertion is thought to depend on the formation of extensive PFO-PFO contacts (Burns et al., 2019; Wade et al., 2015). While further experiments are required to establish whether post-insertion PFO oligomerisation adds monomers to the already inserted arc pore to complete the ring or whether the arc pore facilitates nucleation of a separate oligomer, it is nevertheless tempting to speculate how post-insertion growth may occur. One hypothesis is that the PFO monomer may interact with the inserted arc, for example through initial D4-D4 interactions (Harris et al., 1991), followed by vertical collapse and unfurling of the $\beta$-hairpin (Figure 6-Figure Supplement 3 ). This model is akin to the sequential unfurling of $\beta$-hairpins discussed above and requires considerable conformational flexibility (possibly of the subunits at the edge of the arc). An alternative hypothesis is that incoming PFO monomers may undergo an induced conformational change, e.g. as a result of interacting with the toroidal lipid edge, and subsequently add to the arc pore (Figure 6-Figure Supplement 3). All of our models for post-insertion oligomerisation require PFO to undergo conformational changes, driven through local environmental effects. While this is precisely what drives conventional PFO oligomerisation, characterising the specific details in the context of inserted arcs remains an unresolved question, whereby each hypothesis presented here has puzzling elements but provides the basis for future experimental studies.

As discussed above, insertion can be observed over a wide range of oligomer lengths, ranging from tetramers to full rings. We propose a model in which the transition from arc or ring-shaped prepore to pore is a stochastic process that is controlled by the kinetics of the different steps in 
the pore formation pathway. For example, under conditions where membrane binding is slow (e.g. at low PFO concentrations or $\mathrm{pH}>7$ ), oligomerisation is too slow to complete a full ring before insertion occurs. Since PFO monomers can continue to add after insertion, the final outcome is a ring-shaped pore, regardless of when insertion happened during arc growth. This is consistent with a predominance of ring pores in EM images, even when PFO assembly occurs at low concentrations (Figure 6-Figure Supplement 4). While this model is sufficient to explain the data, additional mechanisms may be operational such as the proposed allosteric trigger for the conformational change upon ring closure (Wade et al., 2015). In addition to arcs and rings, pores can also be formed when two (or more) arcs coalesce into larger structures (Leung et al., 2014; Mulvihill et al., 2015; Ruan et al., 2016; Senior et al., 2021). We expect the relative abundance of these structures to be controlled by kinetics as well. For example, if PFO monomers are depleted from solution during the reaction, arc growth slows down such that coalescence becomes more likely.

Overall, our single-molecule approach enables analysis of all steps leading to PFO pore formation to be deconvoluted and investigated separately, unlike existing ensemble assays. The assay design is simple to modify and enables multiple experimental modalities for different lines of inquiry. Further, the moderate throughput enables statistical power to quantify individual processes within the full assembly pathway. In conjunction with kinetic modelling, this analysis represents a useful method to predict and measure the effects of modification to specific steps in the pathway, with applications in drug development or biotechnology (Johnstone et al., 2021).

\section{Methods}

\section{PFO production and purification}

PFO C459A without signal peptide containing an amino-terminal hexahistidine tag (sequence shown in Supplementary Figure S1; referred to as PFO herein) was expressed in E. coli BL21DE3 cells at $37^{\circ} \mathrm{C}$ using a codon-optimised sequence cloned into the pET-15b plasmid. Cells were lysed in 20 mM HEPES pH 7.5, 150 mM NaCl, 20 mM imidazole, 0.1\% Triton-X 100, protease inhibitor (Sigma) and DNAse I (Sigma). Cell debris was separated from the soluble fraction by centrifugation at $10,000 \mathrm{xg}$ for $30 \mathrm{~min}$ at room temperature. The protein was purified by $\mathrm{Ni}^{2+}$ IMAC chromatography on a HisTrap column (Cytiva). The column was washed using 20 $\mathrm{mM}$ HEPES $\mathrm{pH} 7.5,150 \mathrm{mM} \mathrm{NaCl}, 20 \mathrm{mM}$ imidazole and bound protein eluted using $20 \mathrm{mM}$ HEPES pH 7.5, $150 \mathrm{mM} \mathrm{NaCl}, 500 \mathrm{mM}$ imidazole. Eluted protein was dialysed overnight into 25 $\mathrm{mM}$ HEPES pH 7.5, $150 \mathrm{mM} \mathrm{NaCl}, 2.5 \%$ glycerol and further purified by size exclusion chromatography on a SEC Superdex S200 16/60 column (Cytiva). Peak fractions were concentrated and stored at $-80^{\circ} \mathrm{C}$.

\section{PFO labelling}

PFO was dialysed twice for 1 hour against HBS pH 7.5 (20 mM HEPES, $100 \mathrm{mM} \mathrm{NaCl}$ ) using a Slide-A-Lyzer Mini dialysis capsule (10k MWCO) and the protein concentration (determined 
using the absorbance at $280 \mathrm{nM}$ ) was adjusted to $24 \mu \mathrm{M}$. The labelling reaction was carried out in the dark at room temperature. An aqueous solution of Alexa Fluor 647 (AF647) NHS ester $(2.5 \mathrm{mM})$ was added to a concentration of $120 \mu \mathrm{M}$. The long-wavelength AF647 dye was chosen for PFO as it is less prone to self-quenching than short-wavelength dyes. After a reaction time of $1 \mathrm{~h}$, HisPur Ni-NTA resin $(40 \mu \mathrm{L})$ was added to the mixture. Beads were collected by centrifugation and washed with $\mathrm{HBS} \mathrm{pH} 8(3 \times 50 \mu \mathrm{L})$. After elution with a 1:1 (v:v) mixture of $1 \mathrm{M}$ imidazole $(\mathrm{pH} 7)$ and $\mathrm{HBS}(\mathrm{pH} 7)$ for $10 \mathrm{~min}$, the beads were pelleted by centrifugation and the supernatant containing AF647-PFO was collected. The protein concentration was determined using the Bradford assay. AF647-PFO was analysed by SDS-PAGE with fluorescence densitometry and the degree of labelling was determined to be $\sim 1.5$ fluorophores/PFO by comparison with a labelled protein standard. Analysis of AF647-PFO adsorbed onto a coverslip showed that the majority of PFO molecules ( $60-85 \%$ depending on batch) were singly labelled, while the remainder had more than one label (Supplementary Figure S5). The membrane poration activity of AF647-PFO was essentially the same as that of unlabelled PFO (Figure 1-Figure Supplement 2).

\section{Lipid film preparation and liposome extrusion}

Solutions of cholesterol, 1-palmitoyl-2-oleoyl-glycero-3-phosphocholine (16:0-18:1 POPC) and 1-(12-biotinyl(aminododecanoyl))-2-oleoyl-sn-glycero-3-phosphoethanolamine (12:0 Biotin-18:1 $\mathrm{PE}$ ) in chloroform (10 mg/mL, Avanti Polar Lipids) were mixed in a 55:44:1 molar ratio. The solution was divided into $120 \mu \mathrm{L}$ aliquots in glass vials, dried down under vacuum, overlaid with nitrogen and stored at $-40{ }^{\circ} \mathrm{C}$ until use.

The lipid film was allowed to equilibrate to room temperature, rehydrated in $250 \mu \mathrm{L} \mathrm{HBS} \mathrm{pH} 7$

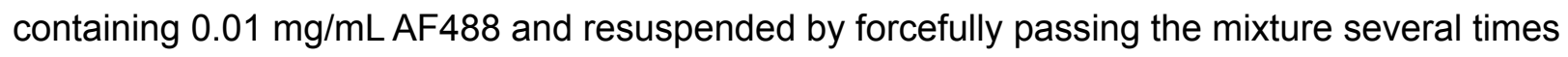
through a 25G needle. The sample was vortexed and subjected to 6 freeze-thaw cycles using liquid nitrogen. Liposomes were extruded using a LiposoFast liposome factory by passing the solution an uneven number of times (21-31) through a $0.22 \mu \mathrm{M}$ polycarbonate membrane filter. The extruded liposomes were passed through a Sephadex G-25 column to remove free dye.

\section{Fabrication and operation of microfluidic flow cells}

Microfluidic fabrication and TIRF assay adapted from (Márquez et al., 2018). Glass coverslips were cleaned by sonication in ethanol for 30 min followed by sonication in $1 \mathrm{M} \mathrm{NaOH}$ for 30 min, rinsed with ultrapure water and dried. PDMS devices for assembly of microfluidic flow cells (channel height $60 \mu \mathrm{m}$, channel width $800 \mu \mathrm{m}$ ) were prepared using standard protocols for soft lithography. After treating the PDMS device and the coverslip with an air plasma inside a plasma cleaner for $5 \mathrm{~min}$, the PDMS device was mounted on the coverslip and the assembled microfluidic flow cell was heated in an oven at $70^{\circ} \mathrm{C}$ for at least $15 \mathrm{~min}$. A second treatment with air plasma was carried out to improve bonding between the glass and the PDMS. The glass surface at the bottom of the microfluidic channels was then modified by adsorption of a co-polymer composed of poly-L-lysine (PLL) and biotinylated poly(ethylene glycol) (PEG) 
(Susos AG, PLL(20)-g[3.4]-PEG(2)/PEG(3.4)-biotin (20\%)). A solution of PLL-PEG-biotin (0.1 $\mathrm{mg} \mathrm{mL}^{-1}$ in PBS) was injected into the flow channels and incubated at room temperature for 5 min followed by flushing the channels with water and drying. The channels were then filled with a solution of streptavidin (Sigma-Aldrich, $0.2 \mathrm{mg} \mathrm{mL}^{-1}$ ) for 15 min and rinsed with $\mathrm{HBS} \mathrm{pH} 7$. The channels were rinsed with isopropanol to remove air bubbles prior to treatment with blocking buffer (20 mM Tris pH 7.5, 2 mM EDTA, $50 \mathrm{mM} \mathrm{NaCl}, 0.03 \%$ NaN3, 0.025\% Tween 20, $0.2 \mathrm{mg} \mathrm{mL}^{-1} \mathrm{BSA}$ ). The microfluidic flow cell was mounted on the microscope stage and connected to tubing. Solutions were pulled through the channels using a syringe pump connected to the outlet tubing and operating in 'withdraw' mode.

\section{TIRF microscopy assays}

AF488-loaded liposomes in HBS (30-100 $\mu \mathrm{L})$ were flowed through the microfluidic channel and captured on the modified coverslip via interaction of the biotinylated lipids with the surface-bound streptavidin. Unbound liposomes were washed out with $50 \mu \mathrm{L}$ of $\mathrm{HBS} \mathrm{pH}$ 7. All experiments were carried out at room temperature unless stated otherwise.

Images were collected on a custom built TIRF microscope based around an ASI-RAMM frame (Applied Scientific Instrumentation) with a Nikon $60 \times$ CFI Apochromat TIRF (1.49 NA) oil immersion objective. Solid State Lasers were incorporated using the NicoLase system (Nicovich et al., 2017). Images were captured on three Photometrics Prime BSI Cameras (Teledyne Photometrics). $250 \mathrm{~mm}$ tube lenses were used to give a field of view of $176 \mu \mathrm{m} \times 176 \mu \mathrm{m}$. Alternatively, single molecule binding images were collected on a TIRF microscope based around a Nikon Eclipse Ti-E2 chassis equipped with a Nikon 100× CFI Apochromat TIRF (1.49 NA) oil immersion objective, solid state lasers for excitation, and a single Photometrics Prime 95B Camera.

\section{Single molecule experiments}

Experiments to measure AF647-PFO monomer binding were acquired as follows. HBS pH 7 (5 $\mathrm{mL})$ containing AF647-PFO $(2.5-15 \mathrm{pM})$ and BSA $(0.01 \mathrm{mg} / \mathrm{mL})$ as a blocking agent was constantly flowing through the channel at a rate of $10 \mu \mathrm{L} / \mathrm{min}$. A total of 432,000 single molecule binding TIRF images were acquired for each independent experiment using a $20 \mathrm{~ms}$ exposure and $100 \mathrm{~mW} 647 \mathrm{~nm}$ laser power at a frame rate of $57 \mathrm{~ms}$ per frame.

Experiments to measure the long-lived species were acquired as follows. HBS pH 7 (5 mL) containing AF647-PFO (10 pM) and BSA $(0.01 \mathrm{mg} / \mathrm{mL})$ was constantly flowing through the channel at a rate of $7 \mu \mathrm{L} / \mathrm{min}$. A total of 2000 images of AF647-PFO were acquired using a 200 $\mathrm{ms}$ exposure, $3 \mathrm{~mW} 647 \mathrm{~nm}$ laser power at a frame rate of $20 \mathrm{~s}$ per frame. In between AF647-PFO images, images of the liposome content dye were captured using 20 ms exposure and $3 \mathrm{~mW} 488 \mathrm{~nm}$ laser power. The liposome images were used to correct for stage drift during acquisition as AF647-PFO binding was too sparse to reliably calculate drift in these experiments. 
Single molecule binding was detected using the Localize program in the Picasso suite (Schnitzbauer et al., 2017) with a minimum net gradient set to 250 for detection of monomers and to 150 for detection of long-lives species. The box size length was set to 9 pixels. Localisations were filtered for particles with a localisation precision of less than 1 in both $x$ and $y$ directions to exclude cases where the background was erroneously detected. Single molecule binding experiments were drift corrected using the redundancy cross-correlation function built into the Render program the Picasso suite with an averaging window size of 10,000 frames. Individual binding events were linked using a maximum link localisation distance of 7 and a maximum number of dark frames of 5 .

\section{PFO concentration titration}

HBS pH 7 containing AF647-PFO (50-500 pM), AF488 (100 nM) as a solution exchange marker and BSA $(0.01 \mathrm{mg} / \mathrm{mL})$ as a blocking agent was constantly flowing through the channel at a rate of $20 \mu \mathrm{L} / \mathrm{min}$. TIRF images were acquired (488 nm laser and 640 laser, $50 \mathrm{~ms}$ exposure time). The total duration of the experiment varied depending on the pore-formation kinetics, lasting between 15 and 240 min, whereby the total number of frames was kept at 180 frames.

\section{PFO wash-out experiment}

HBS (pH 7 and $0.01 \mathrm{mg} / \mathrm{mL}$ BSA) containing AF647-PFO (400 pM) and AF488 (100 nM) as a solution exchange marker was constantly flowing through the channel at a rate of $20 \mu \mathrm{L} / \mathrm{min}$ for 5 minutes. Flow was stopped and inlet tubing was swapped to a $\mathrm{HBS}(\mathrm{pH} 7$ and $0.01 \mathrm{mg} / \mathrm{mL}$ BSA) solution without PFO or dye and flow was resumed at $20 \mu \mathrm{L} / \mathrm{min}$ less than a minute after stoppage. TIRF images were acquired (488 nm laser and 640 laser, $50 \mathrm{~ms}$ exposure time). The total duration of the experiment varied depending on the pore-formation kinetics, lasting between 15 and 240 min, whereby the total number of frames was kept at 180 frames. The temperature was controlled using an OkoLab Bold Line stage top incubator.

\section{Image analysis}

Images were analysed using home-written image analysis software (JIM v4.3, freely available at https://github.com/lilbutsa/JIM-ImmobilizedMicroscopy-Suite). Traces exhibiting loss of the AF488 signal in one step were included in the analysis while multi-step traces, traces without dye release or otherwise uninterpretable traces were excluded from analysis. The time of PFO addition (or PFO wash-out) was detected as an overall increase (or decrease) of the background fluorescence marker. The number of bound AF647-PFO molecules was determined from the ratio of the AF647-PFO fluorescence intensity associated with the liposome to the fluorescence intensity of a single AF647-PFO molecule. The fluorescence intensity of the single fluorophore was determined from the quantal photobleaching step in photobleaching traces of AF647-PFO molecules adsorbed sparsely to the coverslip surface and imaged continuously.

\section{Kinetic models}


The construction of the kinetic models is described in detail in the Appendix.

\section{Acknowledgements}

We thank Quill Bowden, Shaghayegh Baghapour and Chantal Márquez for help and discussion during early stages of assay development. This work was supported by NHMRC Project Grant APP1182212 (TB), ARC Future Fellowship FT150100049 (MD), NHMRC Investigator Grant APP1194263 (MWP), Australian Research Council Grants DP160101874 and DP200102871 (MWP and CJM) and a UNSW International Postgraduate Award (CM). Infrastructure support from the NHMRC Independent Research Institutes Infrastructure Support Scheme and the Victorian State Government Operational Infrastructure Support Program to St. Vincent's Institute are gratefully acknowledged. MWP is an NHMRC Leadership Fellow.

\section{Author contributions:}

Conall McGuinness: Methodology; Formal analysis; Investigation; Writing-Original draft. James Walsh: Conceptualisation; Software; Methodology; Formal analysis; Investigation; Supervision; Writing-Original draft.

Charles Bayly-Jones: Investigation; Formal analysis; Writing-review and editing Michelle Dunstone: Writing-review and editing; Funding acquisition Michelle Christie: Resources; Writing-review and editing Craig Morton: Writing-review and editing Michael Parker: Resources; Writing-review and editing; Funding acquisition Till Böcking: Conceptualisation; Methodology; Writing-original draft; Supervision; Funding acquisition

\section{ORCID IDs}

Conall Mc Guinness: 0000-0002-9733-6699

James Walsh: 0000-0003-0447-2323

Charles Bayly-Jones: 0000-0002-7573-7715

Michelle Dunstone: 0000-0002-6026-648X

Michelle Christie: 0000-0002-7001-3400

Craig Morton: 0000-0001-5452-5193

Michael Parker: 0000-0002-3101-1138

Till Böcking: 0000-0003-1165-3122

\section{References}

Awad MM, Ellemor DM, Boyd RL, Emmins JJ, Rood JI. 2001. Synergistic effects of alpha-toxin and perfringolysin $\mathrm{O}$ in Clostridium perfringens-mediated gas gangrene. Infect Immun 69:7904-7910.

Boyd CM, Parsons ES, Smith RAG, Seddon JM, Ces O, Bubeck D. 2016. Disentangling the roles of cholesterol and CD59 in intermedilysin pore formation. Sci Rep 6:38446.

Burns JR, Morton CJ, Parker MW, Tweten RK. 2019. An Intermolecular m-Stacking Interaction Drives Conformational Changes Necessary to $\beta$-Barrel Formation in a Pore-Forming Toxin. 
MBio 10. doi:10.1128/mBio.01017-19

Christie MP, Johnstone BA, Tweten RK, Parker MW, Morton CJ. 2018. Cholesterol-dependent cytolysins: from water-soluble state to membrane pore. Biophys Rev 10:1337-1348.

Czajkowsky DM, Hotze EM, Shao Z, Tweten RK. 2004. Vertical collapse of a cytolysin prepore moves its transmembrane $\beta$-hairpins to the membrane. The EMBO Journal 23:3206-3215.

Dal Peraro M, van der Goot FG. 2016. Pore-forming toxins: ancient, but never really out of fashion. Nat Rev Microbiol 14:77-92.

Dang TX, Hotze EM, Rouiller I, Tweten RK, Wilson-Kubalek EM. 2005. Prepore to pore transition of a cholesterol-dependent cytolysin visualized by electron microscopy. J Struct Biol 150:100-108.

Dunstone MA, Tweten RK. 2012. Packing a punch: the mechanism of pore formation by cholesterol dependent cytolysins and membrane attack complex/perforin-like proteins. Curr Opin Struct Biol 22:342-349.

Evans JC, Johnstone BA, Lawrence SL, Morton CJ, Christie MP, Parker MW, Tweten RK. 2020. A Key Motif in the Cholesterol-Dependent Cytolysins Reveals a Large Family of Related Proteins. MBio 11. doi:10.1128/mBio.02351-20

Evans JC, Tweten RK. 2021. How protein engineering has revealed the molecular mechanisms of pore-forming toxins. Methods Enzymol 649:47-70.

Feil SC, Rossjohn J, Rohde K, Tweten RK, Parker MW. 1996. Crystallization and preliminary X-ray analysis of a thiol-activated cytolysin. FEBS Lett 397:290-292.

Giddings KS, Zhao J, Sims PJ, Tweten RK. 2004. Human CD59 is a receptor for the cholesterol-dependent cytolysin intermedilysin. Nat Struct Mol Biol 11:1173-1178.

Harris RW, Sims PJ, Tweten RK. 1991. Kinetic aspects of the aggregation of Clostridium perfringens theta-toxin on erythrocyte membranes. A fluorescence energy transfer study. $J$ Biol Chem 266:6936-6941.

Heuck AP, Hotze EM, Tweten RK, Johnson AE. 2000. Mechanism of membrane insertion of a multimeric beta-barrel protein: perfringolysin $O$ creates a pore using ordered and coupled conformational changes. Mol Cell 6:1233-1242.

Heuck AP, Tweten RK, Johnson AE. 2003. Assembly and topography of the prepore complex in cholesterol-dependent cytolysins. J Biol Chem 278:31218-31225.

Hotze EM, Wilson-Kubalek E, Farrand AJ, Bentsen L, Parker MW, Johnson AE, Tweten RK. 2012. Monomer-monomer interactions propagate structural transitions necessary for pore formation by the cholesterol-dependent cytolysins. J Biol Chem 287:24534-24543.

Jacobs T, Darji A, Frahm N, Rohde M, Wehland J, Chakraborty T, Weiss S. 1998. Listeriolysin $\mathrm{O}$ : cholesterol inhibits cytolysis but not binding to cellular membranes. Mol Microbiol 28:1081-1089.

Johnstone BA, Christie MP, Morton CJ, Parker MW. 2021. X-ray crystallography shines a light on pore-forming toxins. Methods Enzymol 649:1-46.

Lawrence SL, Feil SC, Morton CJ, Farrand AJ, Mulhern TD, Gorman MA, Wade KR, Tweten RK, Parker MW. 2015. Crystal structure of Streptococcus pneumoniae pneumolysin provides key insights into early steps of pore formation. Sci Rep 5:14352.

Lawrence SL, Gorman MA, Feil SC, Mulhern TD, Kuiper MJ, Ratner AJ, Tweten RK, Morton CJ, Parker MW. 2016. Structural Basis for Receptor Recognition by the Human CD59-Responsive Cholesterol-Dependent Cytolysins. Structure 24:1488-1498.

Leung C, Dudkina NV, Lukoyanova N, Hodel AW, Farabella I, Pandurangan AP, Jahan N, Pires Damaso M, Osmanović D, Reboul CF, Dunstone MA, Andrew PW, Lonnen R, Topf M, Saibil HR, Hoogenboom BW. 2014. Stepwise visualization of membrane pore formation by suilysin, a bacterial cholesterol-dependent cytolysin. Elife 3:e04247.

Leung C, Hodel AW, Brennan AJ, Lukoyanova N, Tran S, House CM, Kondos SC, Whisstock JC, Dunstone MA, Trapani JA, Voskoboinik I, Saibil HR, Hoogenboom BW. 2017. Real-time visualization of perforin nanopore assembly. Nat Nanotechnol 12:467-473. 
Márquez CL, Lau D, Walsh J, Shah V, McGuinness C, Wong A, Aggarwal A, Parker MW, Jacques DA, Turville S, Böcking T. 2018. Kinetics of HIV-1 capsid uncoating revealed by single-molecule analysis. Elife 7. doi:10.7554/eLife.34772

Morton CJ, Sani M-A, Parker MW, Separovic F. 2019. Cholesterol-Dependent Cytolysins: Membrane and Protein Structural Requirements for Pore Formation. Chem Rev 119:7721-7736.

Mulvihill E, van Pee K, Mari SA, Müller DJ, Yildiz Ö. 2015. Directly Observing the Lipid-Dependent Self-Assembly and Pore-Forming Mechanism of the Cytolytic Toxin Listeriolysin O. Nano Lett 15:6965-6973.

Mutch SA, Fujimoto BS, Kuyper CL, Kuo JS, Bajjalieh SM, Chiu DT. 2007. Deconvolving single-molecule intensity distributions for quantitative microscopy measurements. Biophys $J$ 92:2926-2943.

Nelson LD, Johnson AE, London E. 2008. How interaction of perfringolysin O with membranes is controlled by sterol structure, lipid structure, and physiological low $\mathrm{pH}$ : insights into the origin of perfringolysin O-lipid raft interaction. J Biol Chem 283:4632-4642.

Nicovich PR, Walsh J, Böcking T, Gaus K. 2017. NicoLase-An open-source diode laser combiner, fiber launch, and sequencing controller for fluorescence microscopy. PLoS One 12:e0173879.

Parsons ES, Stanley GJ, Pyne ALB, Hodel AW, Nievergelt AP, Menny A, Yon AR, Rowley A, Richter RP, Fantner GE, Bubeck D, Hoogenboom BW. 2019. Single-molecule kinetics of pore assembly by the membrane attack complex. Nat Commun 10:2066.

Podobnik M, Marchioretto M, Zanetti M, Bavdek A, Kisovec M, Cajnko MM, Lunelli L, Dalla Serra M, Anderluh G. 2015. Plasticity of listeriolysin O pores and its regulation by $\mathrm{pH}$ and unique histidine [corrected]. Sci Rep 5:9623.

Polekhina G, Giddings KS. 2005. Insights into the action of the superfamily of cholesterol-dependent cytolysins from studies of intermedilysin. Proceedings of the.

Ragaliauskas T, Plečkaitytè M, Jankunec M, Labanauskas L, Baranauskiene L, Valincius G. 2019. Inerolysin and vaginolysin, the cytolysins implicated in vaginal dysbiosis, differently impair molecular integrity of phospholipid membranes. Sci Rep 9:10606.

Ramachandran R, Tweten RK, Johnson AE. 2004. Membrane-dependent conformational changes initiate cholesterol-dependent cytolysin oligomerization and intersubunit beta-strand alignment. Nat Struct Mol Biol 11:697-705.

Rosado CJ, Kondos S, Bull TE, Kuiper MJ, Law RHP, Buckle AM, Voskoboinik I, Bird PI, Trapani JA, Whisstock JC, Dunstone MA. 2008. The MACPF/CDC family of pore-forming toxins. Cell Microbiol 10:1765-1774.

Rossjohn J, Feil SC, McKinstry WJ, Tweten RK, Parker MW. 1997. Structure of a cholesterol-binding, thiol-activated cytolysin and a model of its membrane form. Cell 89:685-692.

Rossjohn J, Polekhina G, Feil SC, Morton CJ, Tweten RK, Parker MW. 2007. Structures of perfringolysin $\mathrm{O}$ suggest a pathway for activation of cholesterol-dependent cytolysins. $\mathrm{J} \mathrm{Mol}$ Biol 367:1227-1236.

Ros U, Pedrera L, Garcia-Saez AJ. 2021. Techniques for studying membrane pores. Curr Opin Struct Biol 69:108-116.

Ruan Y, Rezelj S, Bedina Zavec A, Anderluh G, Scheuring S. 2016. Listeriolysin O Membrane Damaging Activity Involves Arc Formation and Lineaction -- Implication for Listeria monocytogenes Escape from Phagocytic Vacuole. PLoS Pathog 12:e1005597.

Sathyanarayana P, Maurya S, Behera A, Ravichandran M, Visweswariah SS, Ayappa KG, Roy R. 2018. Cholesterol promotes Cytolysin A activity by stabilizing the intermediates during pore formation. Proc Natl Acad Sci U S A 115:E7323-E7330.

Schnitzbauer J, Strauss MT, Schlichthaerle T, Schueder F, Jungmann R. 2017. Super-resolution microscopy with DNA-PAINT. Nat Protoc 12:1198-1228. 
Senior MJ, Monico C, Weatherill EE, Gilbert RJ, Heuck AP, Wallace MI. 2021. Single-molecule imaging of cholesterol-dependent cytolysin assembly. bioRxiv. doi:10.1101/2021.05.26.445776

Shatursky O, Heuck AP, Shepard LA, Rossjohn J, Parker MW, Johnson AE, Tweten RK. 1999. The mechanism of membrane insertion for a cholesterol-dependent cytolysin: a novel paradigm for pore-forming toxins. Cell 99:293-299.

Shepard LA, Shatursky O, Johnson AE, Tweten RK. 2000. The mechanism of pore assembly for a cholesterol-dependent cytolysin: formation of a large prepore complex precedes the insertion of the transmembrane beta-hairpins. Biochemistry 39:10284-10293.

Sonnen AF-P, Plitzko JM, Gilbert RJC. 2014. Incomplete pneumolysin oligomers form membrane pores. Open Biol 4:140044.

Spicer BA, Law RHP, Caradoc-Davies TT, Ekkel SM, Bayly-Jones C, Pang S-S, Conroy PJ, Ramm G, Radjainia M, Venugopal H, Whisstock JC, Dunstone MA. 2018. The first transmembrane region of complement component- 9 acts as a brake on its self-assembly. Nat Commun 9:3266.

Tilley SJ, Orlova EV, Gilbert RJC, Andrew PW, Saibil HR. 2005. Structural basis of pore formation by the bacterial toxin pneumolysin. Cell 121:247-256.

Tweten RK. 2005. Cholesterol-dependent cytolysins, a family of versatile pore-forming toxins. Infect Immun 73:6199-6209.

Tweten RK, Parker MW, Johnson AE. 2001. The cholesterol-dependent cytolysins. Curr Top Microbiol Immunol 257:15-33.

van Pee K, Neuhaus A, D'Imprima E, Mills DJ, Kühlbrandt W, Yildiz Ö. 2017. CryoEM structures of membrane pore and prepore complex reveal cytolytic mechanism of Pneumolysin. Elife 6. doi: $10.7554 /$ eLife. 23644

Verherstraeten S, Goossens E, Valgaeren B, Pardon B, Timbermont L, Haesebrouck F, Ducatelle R, Deprez P, Wade KR, Tweten R, Van Immerseel F. 2015. Perfringolysin O: The Underrated Clostridium perfringens Toxin? Toxins 7:1702-1721.

Verherstraeten S, Goossens E, Valgaeren B, Pardon B, Timbermont L, Vermeulen K, Schauvliege S, Haesebrouck F, Ducatelle R, Deprez P, Van Immerseel F. 2013. The synergistic necrohemorrhagic action of Clostridium perfringens perfringolysin and alpha toxin in the bovine intestine and against bovine endothelial cells. Vet Res 44:45.

Vögele M, Bhaskara RM, Mulvihill E, van Pee K, Yildiz Ö, Kühlbrandt W, Müller DJ, Hummer G. 2019. Membrane perforation by the pore-forming toxin pneumolysin. Proc Natl Acad Sci $U$ S A 116:13352-13357.

Wade KR, Hotze EM, Kuiper MJ, Morton CJ, Parker MW, Tweten RK. 2015. An intermolecular electrostatic interaction controls the prepore-to-pore transition in a cholesterol-dependent cytolysin. Proc Natl Acad Sci U S A 112:2204-2209.

Wade KR, Lawrence SL, Farrand AJ, Hotze EM, Kuiper MJ, Gorman MA, Christie MP, Panjikar S, Morton CJ, Parker MW, Tweten RK. 2019. The Structural Basis for a Transition State That Regulates Pore Formation in a Bacterial Toxin. MBio 10. doi:10.1128/mBio.00538-19 
bioRxiv preprint doi: https://doi.org/10.1101/2021.10.19.464937; this version posted October 19, 2021. The copyright holder for this preprint (which was not certified by peer review) is the author/funder. All rights reserved. No reuse allowed without permission.

A cross-section

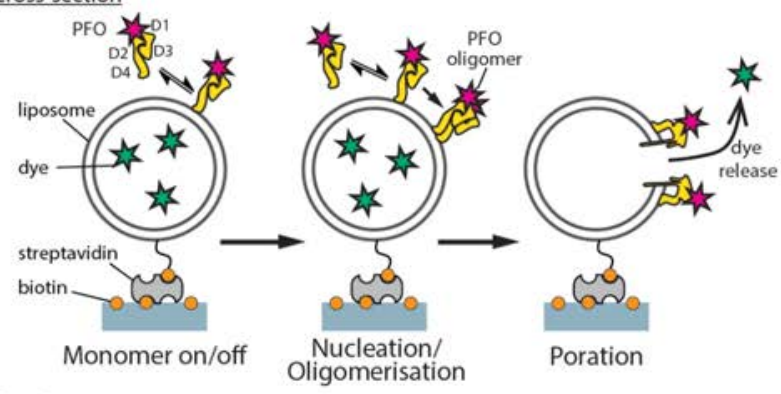

B
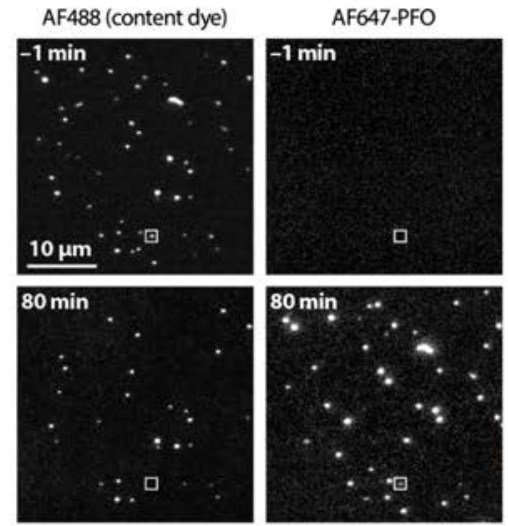

$\mathrm{E}$

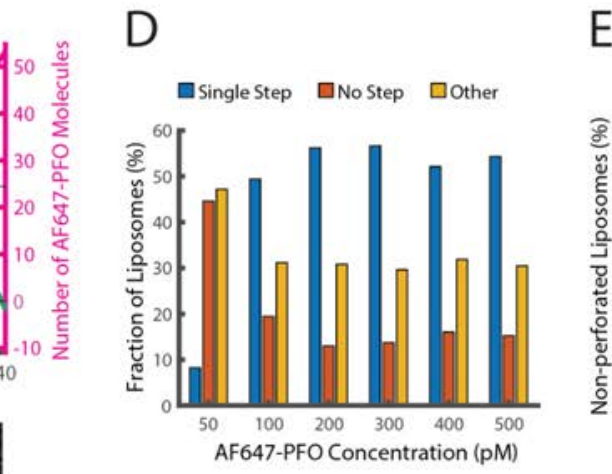

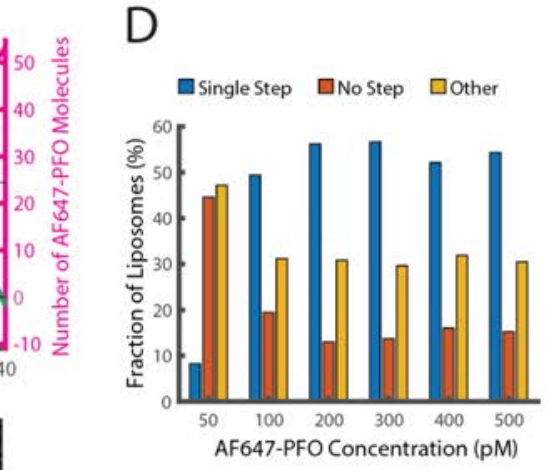

Overlay
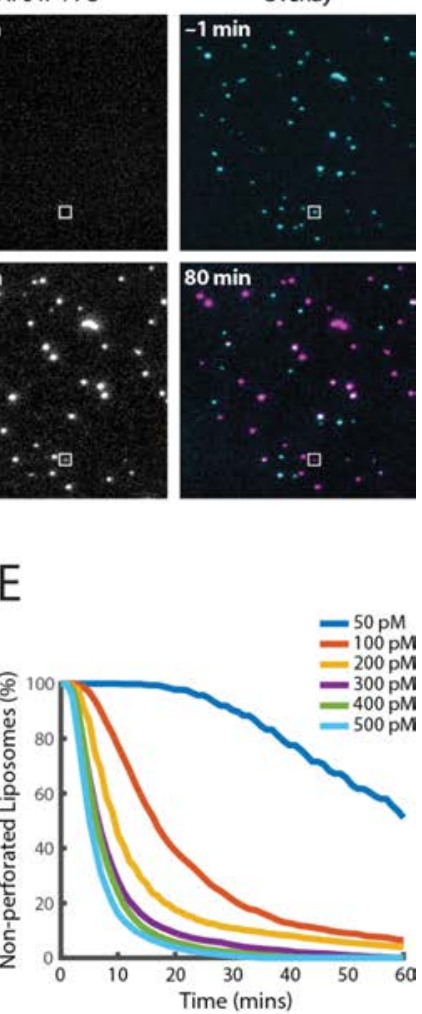

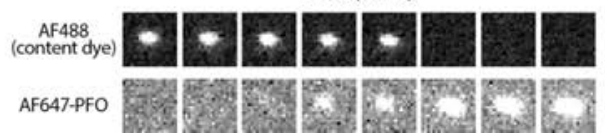

Figure 1. PFO pore formation assay. (A) Schematic of the TIRF assay to measure the PFO assembly and membrane pore formation. Liposomes loaded with AF488 as a content marker are bound to a coated glass coverslip at the bottom of a microfluidic device. AF647-labelled PFO injected into the flow channel reversibly binds to the liposome, assembles into an oligomer and ultimately forms an open arc or ring pore releasing the encapsulated dye. (B) TIRF images $(400 \times 400$ pixel region) from a pore formation time series before $(-1 \mathrm{~min})$ and after (80 $\mathrm{min}$ ) addition of $100 \mathrm{pM}$ AF647-PFO to immobilised liposomes showing the AF488 channel (left), the AF647-PFO channel (middle) and an overlay of both channels (right; AF488 in cyan and AF647-PFO in magenta). (C) Example fluorescence intensity traces recorded at the location of the single liposome (marked with the white box in panel B) in the content dye (blue-green) and AF647-PFO (magenta) channels. Dye release pinpoints the time of membrane permeabilisation. Snapshots of the corresponding liposome in both channels are shown below the traces. Additional example traces are shown in Figure 1-Figure Supplement 3. (D) Fraction of liposomes with content dye traces classified on the basis of step fitting as (1) single step, (2) no dye loss ( $<25 \%$ decrease in intensity) or (3) other release profiles (includes traces with multiple steps or incomplete dye loss). Example traces for each of these classes are shown in Figure 1-Figure Supplement 3.

(E) Distributions of single-step dye release times from liposomes at AF647-PFO concentrations between 50-500 pM. 
bioRxiv preprint doi: https://doi.org/10.1101/2021.10.19.464937; this version posted October 19, 2021. The copyright holder for this preprint (which was not certified by peer review) is the author/finder, All rights reserved. No reusetallowed without permission.

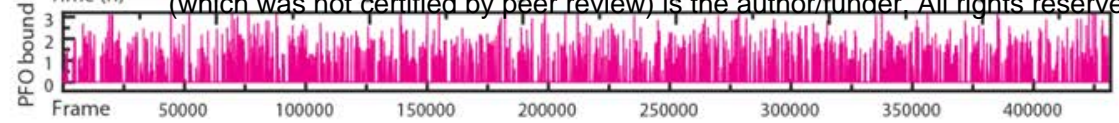

B

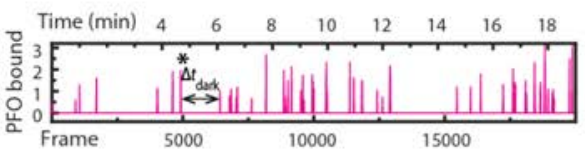

C
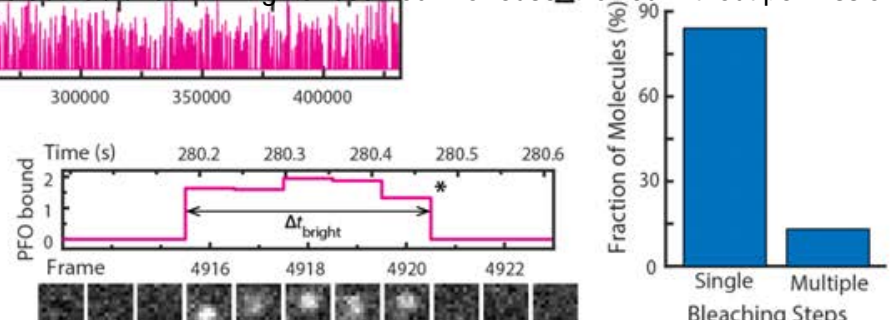

$\mathrm{D}$
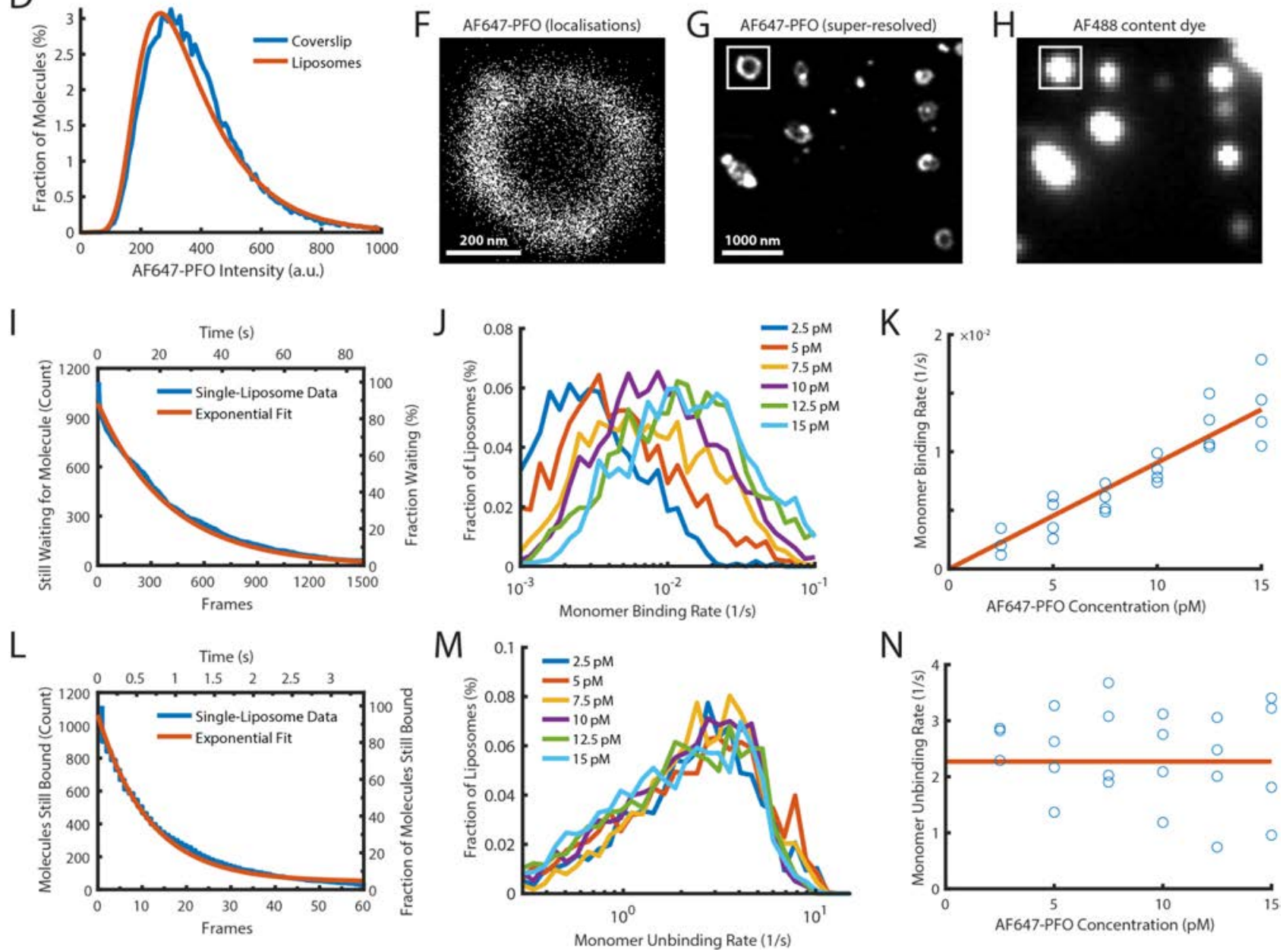

Figure 2. Single-molecule PFO binding to liposomes is short-lived. (A-C) Trace of single AF647-PFO molecules detected at the location of a single liposome from a time series (432000 frames at 17.5 frames per second) acquired in the presence of 10 pM AF647-PFO. (A) Entire trace (6.8 hours). (B) Expanded view of the first $20 \mathrm{~min}$. The double arrow indicates the waiting time $\left(\Delta t_{\text {dark }}\right)$ between the peak marked with an asterisk and the subsequent peak. (C) Expanded view of the peak marked with an asterisk in B. The double arrow indicates the duration ( $\Delta t_{\text {bright }}$ ) of a AF647-PFO molecule on the liposome. Snapshots of the molecule detected in 5 successive frames are shown below the trace. (D) Intensity distribution of AF647-PFO molecules bound to liposomes (orange line) or immobilised directly on a glass coverslip (blue). (E) Photobleaching analysis of AF647-PFO molecules immobilised on a glass coverslip. The majority (84\%) of AF647-PFO bleached in a single step confirming that they are monomeric. (F) Map of $x / y$-localisations determined by point-spread function fitting of single AF647-PFO molecules transiently binding to the same single liposome as in A-C. (G) Super-resolved image showing liposome outlines reconstructed from single AF647-PFO localisations. The reconstruction inside the box corresponds to the localisations shown in C. (H) Diffraction-limited TIRF image of the AF488 content dye of the same area as shown in D. An overlay of a larger region of the field of view in both channels is shown in Figure 2-Figure Supplement 1. (I-K) Analysis of AF647-PFO monomer binding. (I) Distribution of dwell times in the bound state (blue line) extracted from the trace shown in A. An exponential fit of the curve is shown in 


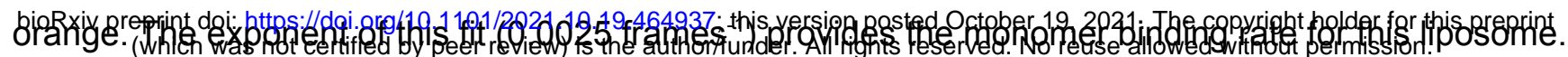
(J) Distributions of binding rates from all liposomes in the field of view measured at different AF647-PFO concentrations. $(\mathrm{K})$ Monomer binding rates increase linearly with AF647-PFO concentration. Each data point represents the median binding rate determined from the corresponding distribution recorded in an independent TIRF time series ( 3 time series at $2.5 \mathrm{pM}, 4$ time series per concentration between 5-15 $\mathrm{pM})$. The orange line represents a linear fit of the data, whereby the slope of the line provides an estimate of the monomer binding rate constant. (L-N) Analysis of AF647-PFO monomer unbinding. (L) Distribution of waiting times between molecules (blue line) extracted from the trace shown in A. An exponential fit of the curve is shown in orange. The exponent of this fit $\left(0.086\right.$ frames $\left.^{-1}\right)$ is corrected for photobleaching $\left(0.0517\right.$ frames $\left.^{-1}\right)$ and then taken as the monomer unbinding rate $\left(0.6 \mathrm{~s}^{-1}, 0.343\right.$ frames $\left.^{-1}\right)$ for this liposome. (M) Distribution of unbinding rates from all liposomes in the field of view measured at different AF647-PFO concentrations. (N) Monomer unbinding is independent of concentration. Each data point represents the median unbinding rate determined from the corresponding distribution recorded in the same 23 experiments as in $\mathrm{K}$. The orange line represents the median of all experiments. 
A
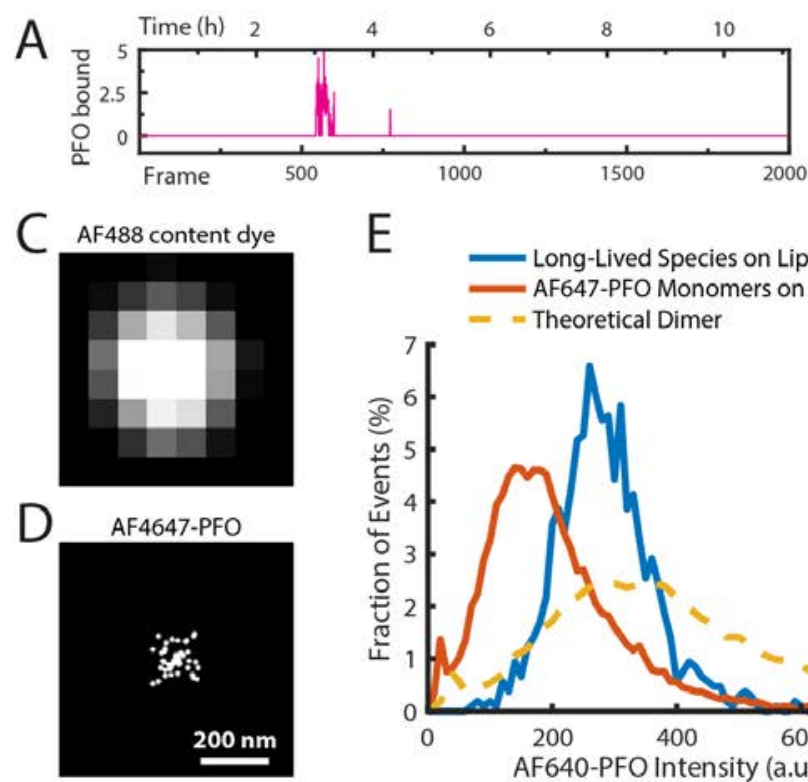
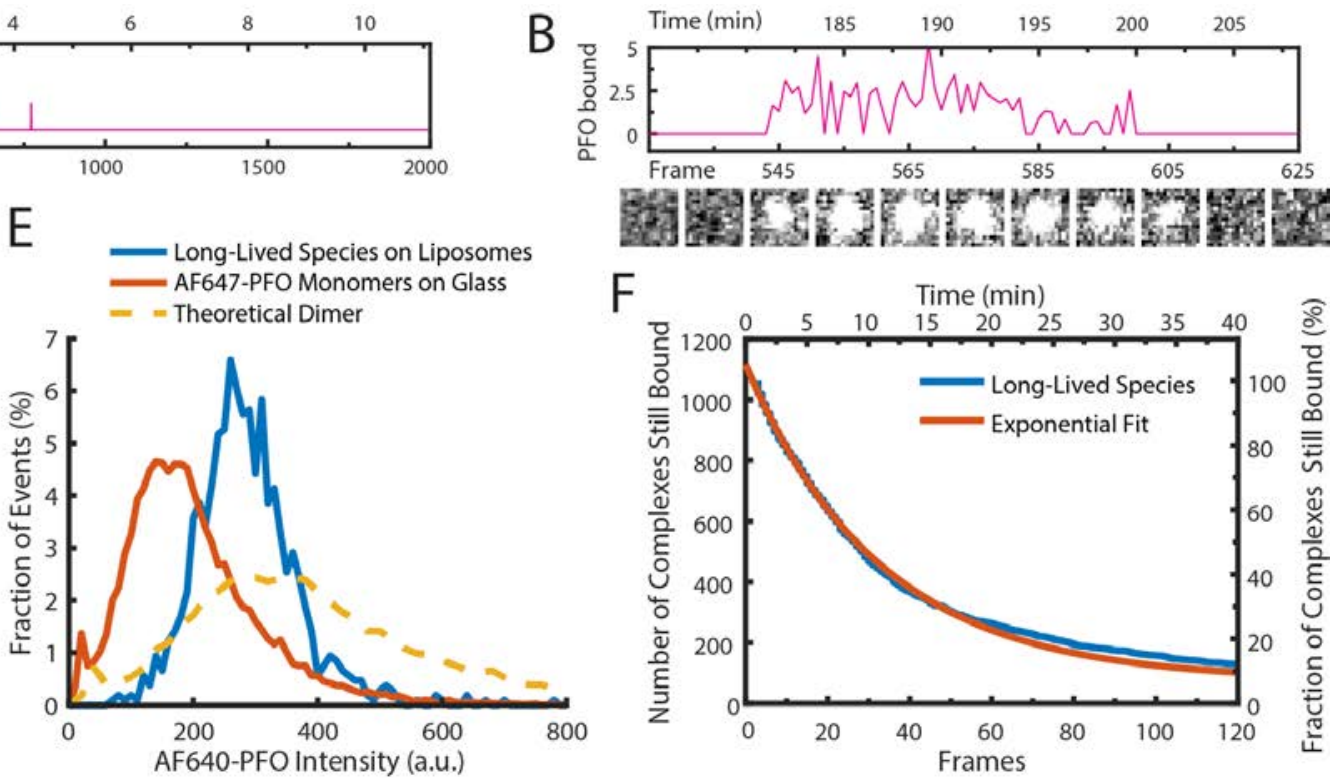

$\mathrm{F}$

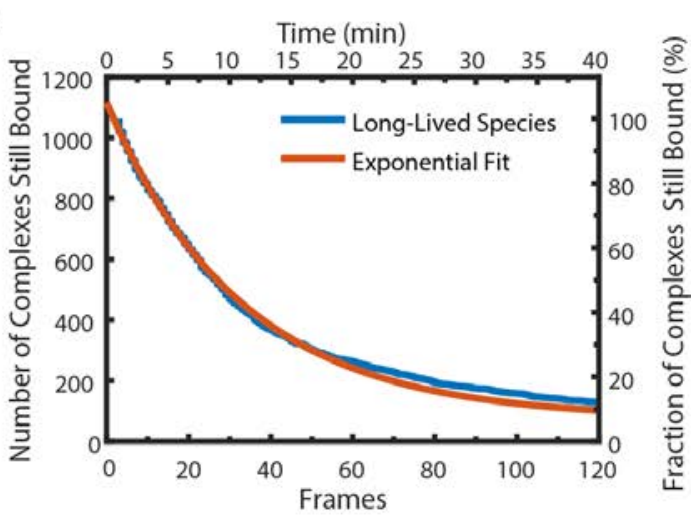

Figure 3. AF647-PFO dimers are able to stably bind to liposomes. (A,B) Trace containing a long-lived state detected at the location of a single liposome from a time series (2000 frames at 3 frames per minute) acquired in the presence of $10 \mathrm{pM} \mathrm{AF647-PFO.} \mathrm{Under} \mathrm{these} \mathrm{conditions,} \mathrm{detection} \mathrm{of} \mathrm{short-lived} \mathrm{monomers} \mathrm{is} \mathrm{rare.} \mathrm{(B)}$ Expanded view of the peak in A, with corresponding snapshots (averaged over a window of 5 frames) shown below. (C,D) Diffraction-limited TIRF image of the AF488 content dye (C) and corresponding map of $\mathrm{x} / \mathrm{y}$-localisations of the AF647-PFO species (D) detected during the peak in A. (E) Intensity distributions of long-lived states on liposomes (blue) and of immobilised AF647-PFO monomers on a glass (orange). The yellow dashed line is the theoretical distribution for AF647-PFO dimers calculated from the monomer intensity distribution. (F) Distribution of dwell times in the long-lived bound state (blue line) extracted from 1063 events acquired in 5 experiments. An exponential fit with a decay constant of 0.03 frame $^{-1}$ is shown in orange. After correction for photobleaching $\left(0.0133\right.$ frame $\left.^{-1}\right)$, this analysis gives a dimer disappearance rate of $D_{\text {off }}=0.052$ $\min ^{-1}$. 
bioRxiv preprint doi: https://doi.org/10.1101/2021.10.19.464937; this version posted October 19, 2021. The copyright holder for this preprint (which was not certified by peer review) is the author/funder. All rights reserved. No reuse allowed without permission.

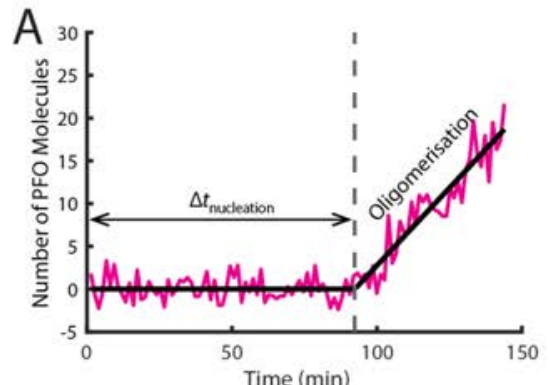

$\mathrm{D}$

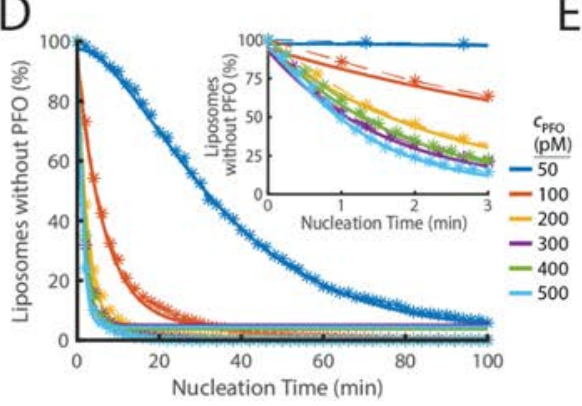

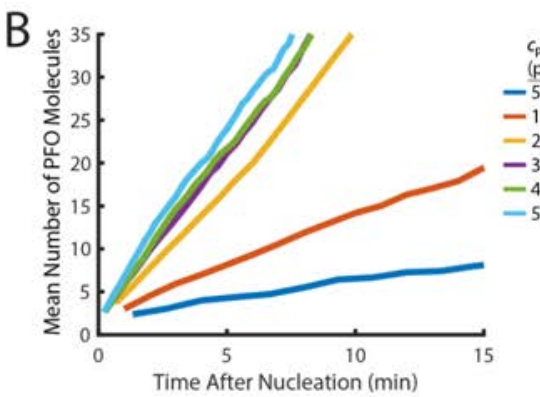

$\mathrm{E}$

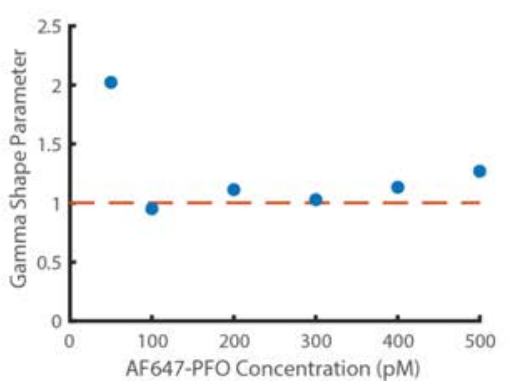

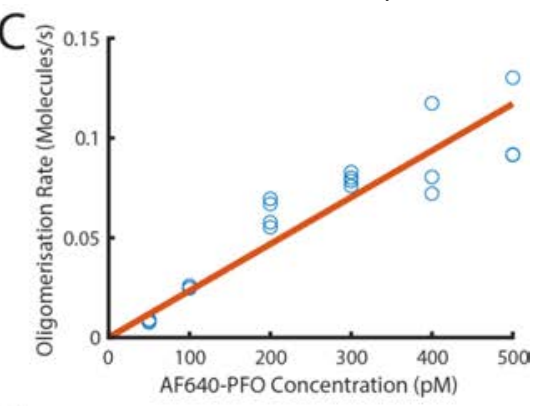

$\mathrm{F}$

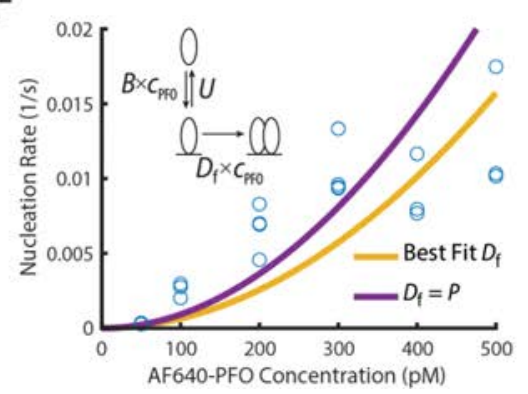

Figure 4. PFO nucleation and oligomerisation. (A) AF647-PFO intensity trace of the early stages of PFO assembly showing fluctuations around the baseline (before nucleation) and signal increase due to oligomerisation (after nucleation). The slope of the linear fit line provides the oligomerisation rate. The nucleation time ( $\left.\Delta t_{\text {nucleation }}\right)$ is defined at the time where the oligomerisation fit line intersects the baseline. (B) The mean of all traces after they have been aligned to their time of nucleation displays a linear increase in intensity resulting from oligomerisation. (C) Oligomerisation rate as a function of AF647-PFO concentration; each data point represents the median oligomerisation rate determined in an independent PFO pore formation experiment ( 3 experiments for 50 pM, 100 pM, 400 pM, 500 pM; 4 experiments for 200 pM, 300 pM). The slope of the linear fit (orange line) provides the oligomerisation rate constant $P=0.23 \mathrm{nM}^{-1} \mathrm{~s}^{-1}$. (D) Experimental nucleation time distributions measured at different concentrations are represented by dashed lines with stars. Each distribution is an average of at least three experiments. Gamma distributions fitted to the experimental data are represented by solid lines. Inset shows a zoom in of the first 3 minutes (E) Gamma shape parameter vs. AF647-PFO concentration determined from the fits of the nucleation time distributions in (D). The gamma distribution fit to the nucleation times for 100-500 pM yielded a shape parameter of approximately 1 (mean of 1.09), consistent with a single rate-limiting step. A shape parameter of 1 is the equivalent to an exponential fit. In contrast, fitting the $50 \mathrm{pM}$ data required a shape parameter of 2.02, suggesting an additional step becomes rate-limiting at this concentration. (F) Nucleation rate as a function of AF647-PFO concentration. The value for $D_{f}$ was obtained by fitting ("Best fit", yellow line), giving a value of $0.16 \mathrm{nM}^{-1} \mathrm{~s}^{-1}$. Alternatively, the value for $D_{f}$ was assumed to be identical to the oligomerisation rate constant $P=0.16 \mathrm{nM}^{-1} \mathrm{~s}^{-1}$ (purple line). Inset: Kinetic model for predicting the concentration dependence of the nucleation rate, where $B$ is the monomer binding rate constant, $U$ is the monomer unbinding rate, $D_{f}$ is the dimerisation rate constant and $c_{\mathrm{PFO}}$ is the PFO concentration. The values for $B$ and $U$ were obtained from the single-molecule binding experiments in Figure 2 . 
bioRxiv preprint doi: https://doi.org/10.1101/2021.10.19.464937; this version posted October 19, 2021. The copyright holder for this preprint (which was not certified by peer review) is the author/funder. All rights reserved. No reuse allowed without permission.
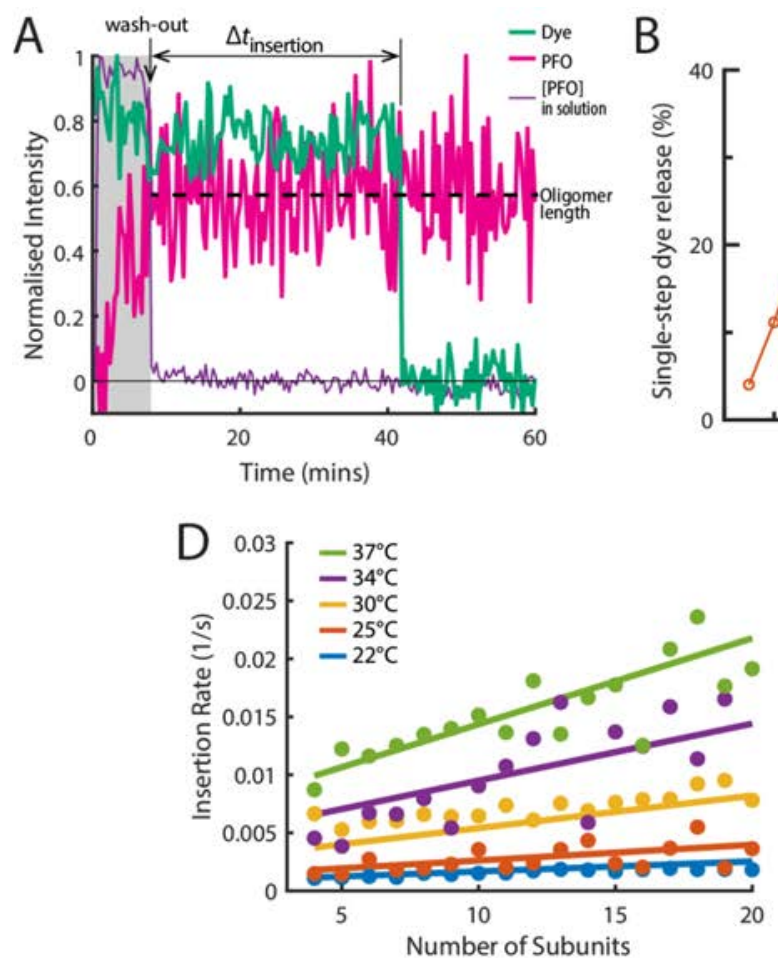

B

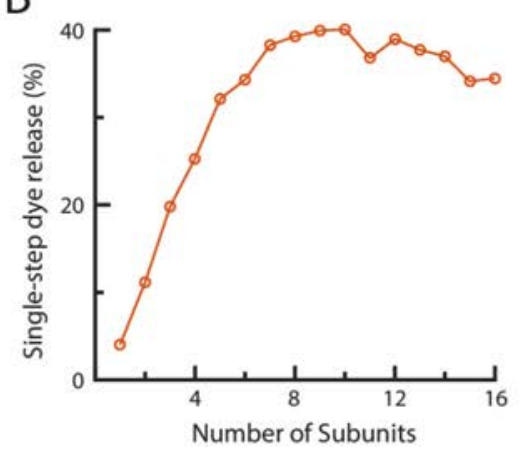

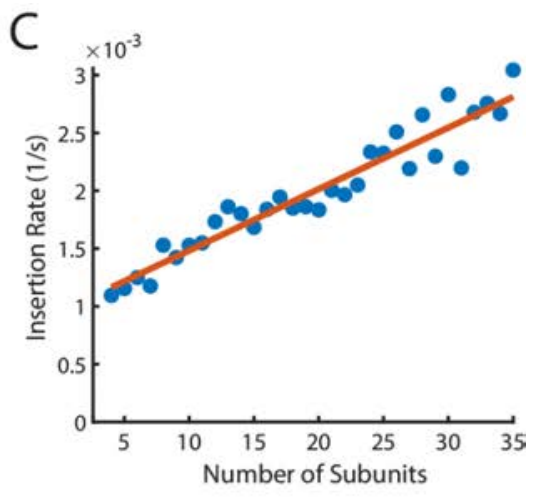

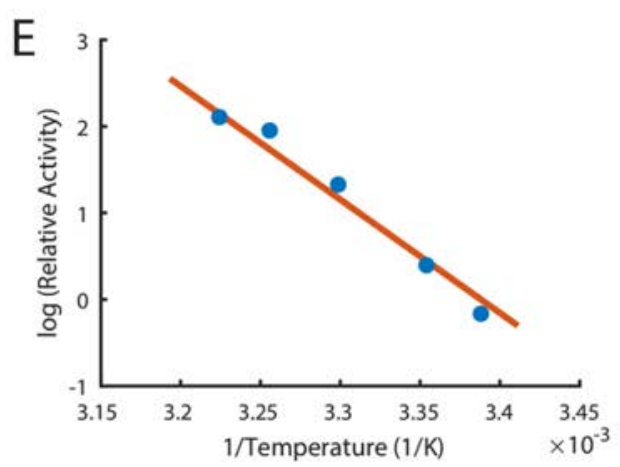

Figure 5. The energetics of PFO oligomer membrane insertion depend on oligomer length. (A) Example trace of the AF647-PFO washout experiment. The purple line represents the background intensity of AF647-PFO in solution; the blue-green line represents the intensity of the AF488 content dye marker; the magenta line represents the AF647-PFO intensity on the liposome. The insertion time is determined as the waiting time between loss of the background signal (wash-out) and loss of content dye signal (pore opening). The AF647-PFO intensity at the time of wash-out (dotted line) was used to determine the oligomer length. (B) Pore opening efficiency (determined as the fraction of liposomes that release the content dye in a single step) as a function of the number of subunits. (C) Insertion rate (determined from the exponential fit of the corresponding insertion time distribution) as a function of the number of subunits with line of best fit. (D) Insertion rate as a function of the number of subunits determined at different temperatures. The fit lines were obtained by a global fit of the data (weighted on the basis of the number of liposome observations), whereby the only free parameter of the fit is proportional to the activation energy. (E) Arrhenius plot of the relative activity of insertion (blue data points) obtained from the slopes of local fits of the data in $\mathrm{D}$. The slope of the orange line is obtained from the parameter value obtained from the global fit in D. 
A bioRxiv preprint doi: https://doi.org/10.1101/2021.10.19.464937; this version posted October 19, 2021. The copyright holder for this preprint

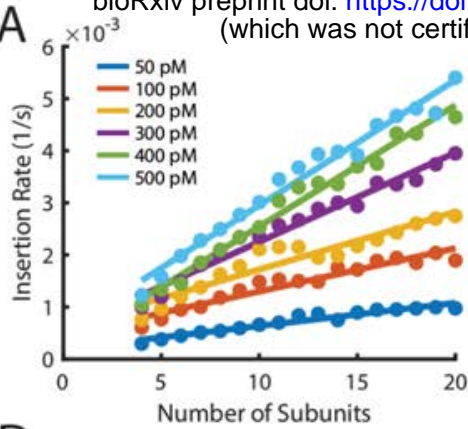

D

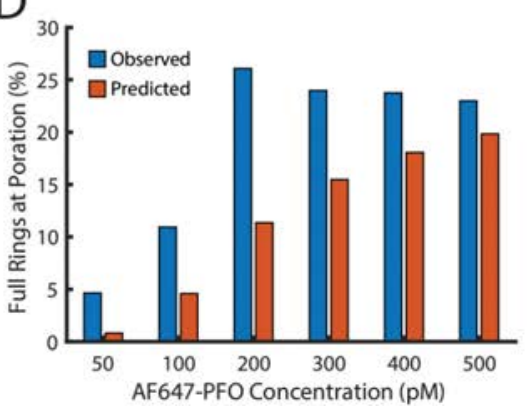

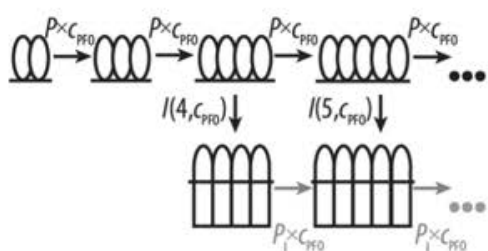

$\mathrm{E}$

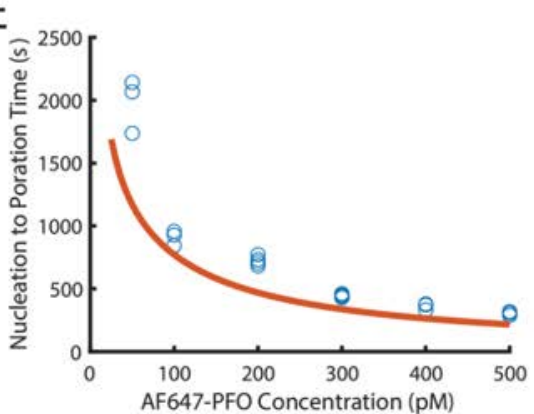

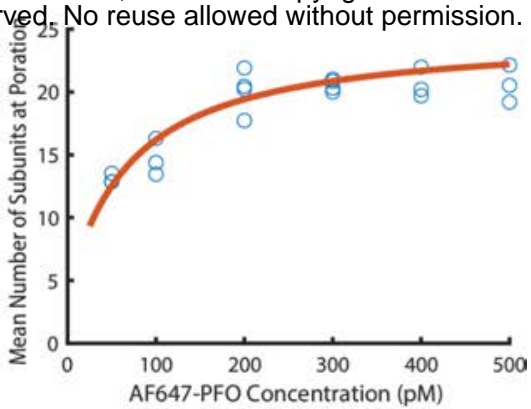

$\mathrm{F}$

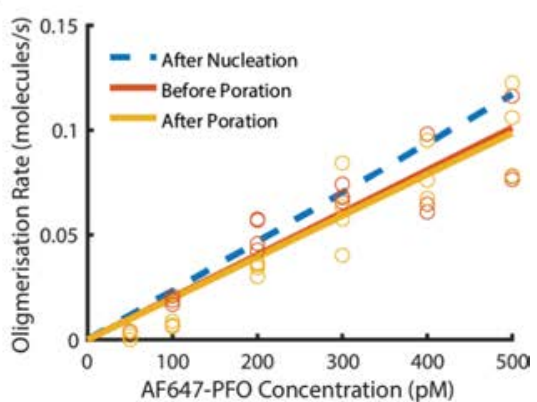

Figure 6. PFO insertion kinetics during continuous growth. (A) The insertion rate of continuously growing PFO arcs depends on the number of subunits in the arc $(n)$ and the concentration of AF647-PFO in solution $\left(c_{\mathrm{pfo}}\right)$ and is given by: $I\left(n, c_{\mathrm{pfo}}\right)=I_{0}+n\left(I_{\mathrm{g} 0}+c_{\mathrm{pfo}} \times I_{\mathrm{gc}}\right)$ where $I_{0}, I_{\mathrm{g} 0}$ and $I_{\mathrm{gc}}$ are the kinetic parameters for insertion (See Figure 6 Figure Supplement 1). (B) Kinetic model used to predict oligomer lengths and insertion kinetics.

Dimerisation is the committed step for continued oligomerisation. Arcs with at least 4 subunits can insert into the membrane (or continue to grow), whereby the insertion rate increases with oligomer length (Figure 6A). The parameter values were obtained from experiment and are shown in Table 1. (C) Mean number of subunits at the time of poration as a function of AF647-PFO concentration. Each data point is the mean from an independent experiment. The orange line shows the prediction from the model in B. (D) Fraction of complete rings formed at the time of poration. (E) Time from nucleation to poration as a function of time. The orange line shows the prediction from the model in B. (F) Oligomerisation kinetics are the same before (yellow) and after (orange) poration. The fit line of the oligomerisation kinetics measured after nucleation (Figure 3D) is shown as a dashed blue line. 
bioRxiv preprint doi: https://doi.org/10.1101/2021.10.19.464937; this version posted October 19, 2021. The copyright holder for this preprint (which was not certified by peer review) is the author/funder. All rights reserved. No reuse allowed without permission.
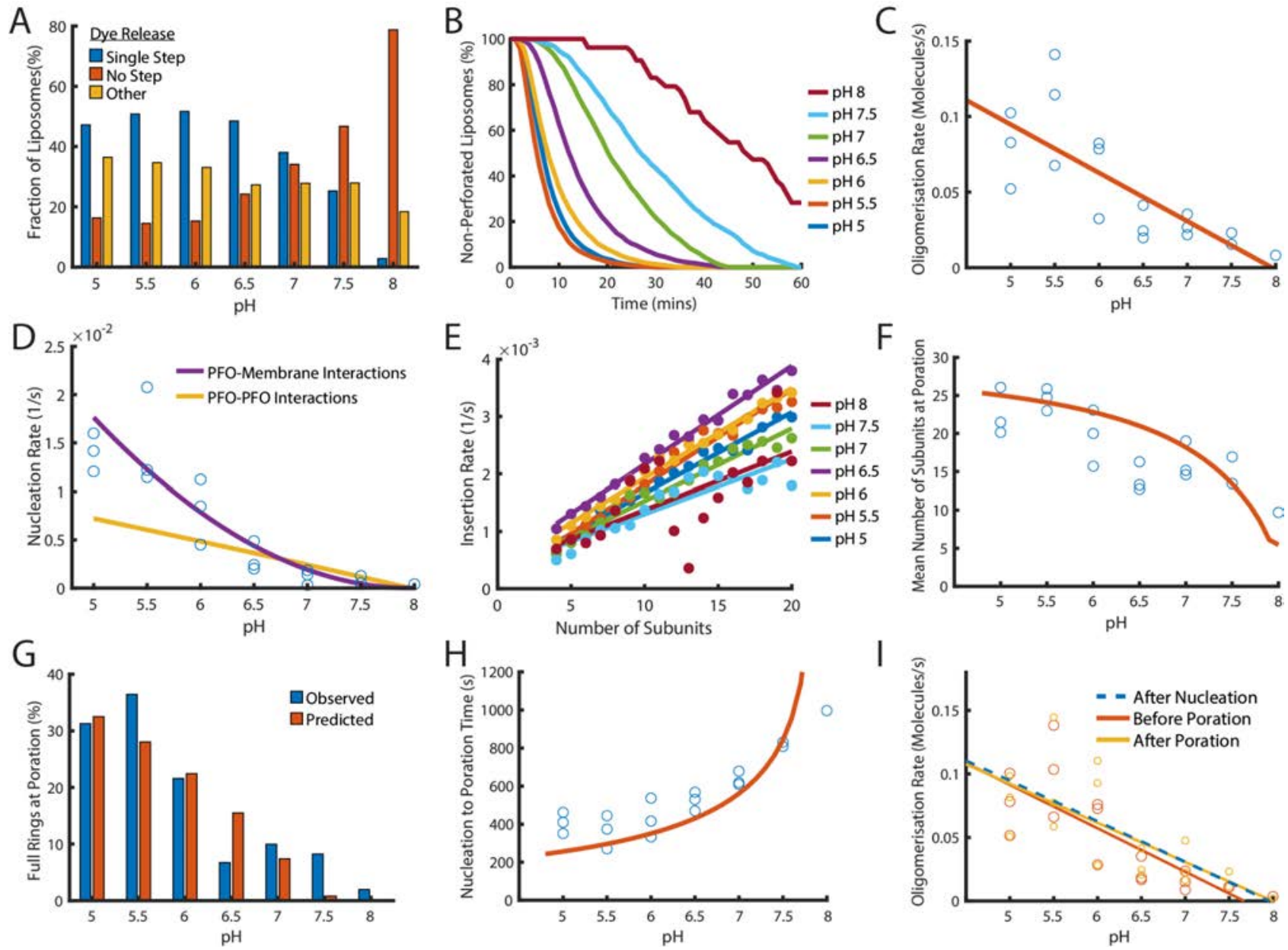

Figure 7. Dependence of PFO pore formation on pH. Dual-colour TIRF pore formation experiments were carried out with 200 pM AF647-PFO (A) AF647-PFO pore formation activity at 200 pM decreases with increasing $\mathrm{pH}$ resulting in a higher proportion of liposomes with no step. Fraction of liposomes with content dye traces classified as (1) single step, (2) no/little dye loss or (3) other. (B) The overall rate of pore formation decreases with increasing $\mathrm{pH}(\mathbf{C})$ The oligomerisation rate decreases with increasing $\mathrm{pH}$. The dependence is heuristically fit with a straight line (D) The nucleation rate decreases with increasing $\mathrm{pH}$. The $\mathrm{pH}$ dependence of oligomerisation observed in (B) could result from $\mathrm{pH}$ affecting either the membrane binding kinetics of monomeric PFO, or the lateral interaction between membrane-bound PFO monomers. These two scenarios give rise to different predicted nucleation dependence curves shown in purple and yellow, respectively. (E) Changing $\mathrm{pH}$ does not have a significant effect on the insertion kinetics (F) The mean insertion length decreases with increasing $\mathrm{pH}$. This dependence is predicted by taking the oligomerisation rate from (C), suggesting that $\mathrm{pH}$ does not affect insertion kinetics. (G) The percentage of full pores at time of poration decreases with increasing $\mathrm{pH}$. The dependence is predicted using the oligomerisation rate from (B). $(\mathbf{H})$ The nucleation to insertion time increases with increasing $\mathrm{pH}$. The dependence is predicted using the oligomerisation rate from (B). (I) Oligomerisation kinetics at different $\mathrm{pH}$ are the same before (Blue) and after (Red) poration. 


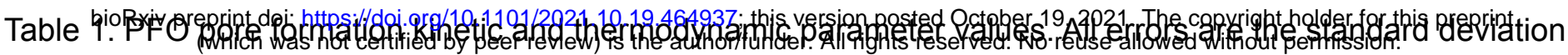
of independent measurements (see Appendix)

\begin{tabular}{|l|l|l|l|}
\hline Parameter & Symbol & Value & Units \\
\hline Monomer binding rate constant & $B$ & $0.9 \pm 0.23$ & $\mathrm{nM}^{-1} \mathrm{~s}^{-1}$ \\
\hline Monomer unbinding rate & $U$ & $2.27 \pm 0.81$ & $\mathrm{~s}^{-1}$ \\
\hline Dimer formation rate constant & $D_{f}$ & $0.16 \pm 0.19$ & $\mathrm{nM}^{-1} \mathrm{~s}^{-1}$ \\
\hline Dimer dissociation rate & $D_{r}$ & $(8.6 \pm 3.4) \times 10^{-4}$ & $\mathrm{~s}^{-1}$ \\
\hline Oligomerisation rate constant & $P$ & $0.23 \pm 0.028$ & $\mathrm{nM}^{-1} \mathrm{~s}^{-1}$ \\
\hline Insertion rate (at $\left.22^{\circ} \mathrm{C}\right)$ & & & $(5.0 \pm 2.4) \times 10^{-4}$ \\
\hline & $I_{0}$ & $(4.0 \pm 1.6) \times 10^{-5}$ & $\mathrm{~s}^{-1}$ \\
\hline Activation energy for insertion & $E_{g 0}$ & $(1.8 \pm 0.63) \times 10^{-19}$ & $\mathrm{~J}$ \\
\hline & $I_{g c}$ & $(4.25 \pm 1.45) \times 10^{-10}$ & $\mathrm{nM}^{-1} \mathrm{~s}^{-1}$ \\
\hline
\end{tabular}

